Journal of Business \& Economic

Statistics

프를 픈

\section{Journal of Business \& Economic Statistics}

ISSN: 0735-0015 (Print) 1537-2707 (Online) Journal homepage: http://www.tandfonline.com/loi/ubes20

\title{
Testing Nowcast Monotonicity with Estimated Factors
}

\section{Jack Fosten \& Daniel Gutknecht}

To cite this article: Jack Fosten \& Daniel Gutknecht (2018): Testing Nowcast Monotonicity with Estimated Factors, Journal of Business \& Economic Statistics, DOI: 10.1080/07350015.2018.1458623

To link to this article: https://doi.org/10.1080/07350015.2018.1458623

View supplementary material $\circlearrowright$

Accepted author version posted online: 04 Apr 2018.

Submit your article to this journal

山 Article views: 13

Q View related articles $\widetilde{ }$

View Crossmark data \lceil 


\title{
Testing Nowcast Monotonicity with Estimated Factors*
}

\author{
Jack Fosten ${ }^{\dagger} \quad$ Daniel Gutknecht ${ }^{\ddagger}$
}

March 15, 2018

\begin{abstract}
This paper proposes a test to determine whether 'big data' nowcasting methods, which have become an important tool to many public and private institutions, are monotonically/mpioving as new information becomes available. The test is the first to formalise existing evaluatic n procedures from the nowcasting literature. We place particular emphasis on models involving estimateu factors, since factor-based methods are a leading case in the high-dimensional empi-ical nswcasting literature, although our test is still applicable to small-dimensional set-ups like bridge equations and MIDAS models. Our approach extends a recent methodology for testing many moment inequalities to the case of nowcast monotonicity testing, which allows the number of ineanalities to grow with the sample size. We provide results showing the conditions under which both parameter estimation error and factor estimation error can be accommodated in this high dimensional setting when using the pseudo out-of-sample approach. The finite sample nerformance of our test is illustrated using a wide range of Monte Carlo simulations, and we conclude with an empirical application of nowcasting U.S. real gross domestic product (GDP) growth and five GDP sub-components. Our test results confirm monotonicity for all but ore sub component (government spending), suggesting that the factor-augmented model may be missperified for this GDP constituent.
\end{abstract}

JEL Classification: $\mathrm{C} 12, \mathrm{C} 2 \mathrm{2}, \mathrm{C} 52, \mathrm{C} 53$

Keywords: Nowcastino, Factor Models, Moment Inequalities, Bootstrap

${ }^{*}$ We are grateful to the editor Todd Clark, an Associate Editor, and two anonymous referees, as well as to Valentina Corradi, Rob Sollis, Ana Galvão, and Matthias Hartmann, and seminar participants at Newcastle University Business School, the 2016 CMF-CEF Seville meeting, the SNDE 2017 meeting in Paris, Lund University, Mannheim University and the 70th European Meeting of the Econometric Society in Lisbon for insightful comments and discussions.

${ }^{\dagger}$ Department of Economics, University of East Anglia, Norwich, NR4 7TJ, UK. E-mail address: j.fosten@uea.ac.uk.

${ }^{\ddagger}$ Department of Economics, University of Mannheim, 68131 Mannheim, Germany. E-mail address: Daniel.Gutknecht@gmx.de. 


\section{ACCEPTED MANUSCRIPT}

\section{Introduction}

Nowcasting methods have been introduced as a way to provide timely predictions of the current state of the economy. This is important as key policy variables such as GDP are typically published at a low frequency and with a significant publication lag. These methods address important irregularities of macroeconomic datasets, specifically the problems of mixed frequency predictors and the so-cailea 'ragged edge' problem of the staggered release of data series by different statistical agencies. Pne of the most desirable features of nowcasting methods is that they can be used to revise predictions of the target variable multiple times per quarter, as soon as any new piece of information beromes available. However, while there has been a significant body of literature devoted to developing new nowcasting methods, there has been very little work on how to formally assess their performance

Recent empirical nowcasting studies indicate that the evaluative criteria of nowcast methods have moved in a different direction to the traditional forecast evaluation testing iter ature which dates back to Diebold and Mariano (1995) and West (1996). Since a major advantage of nowcasting methods is to use big data in making very timely predictions of the economy, as noted in Bańbura et al. (2013), it is not as relevant to assess whether a noweasting method is capable of outperforming naïve benchmarks like autoregressions, or surveys of professional forecasters, which potentially only produce one prediction per quarter. As such, it has become common for nowcasters to check whether nowcasts based on a particular methou are monotonically improving as new information is added, using statistics like mean squared ferecast) eror (MSFE). Nowcast monotonicity has been used as an evaluative criterion at policy-rnakjing institutions such as the Atlanta Fed and in empirical papers dating back to Giannone et al. (2008) and many more recently (see for example Bańbura et al., 2013. Aastveit et al., 2014, Iuciani and Ricci, 2014, Marcellino et al., 2016, Knotek and Zaman, 2017, Bragoli and Fosten, 2018).

Nowcast monotonicity is also an interesting evaluative criterion as it has links to the literature on forecester rationality. In the context of long-horizon forecasting, Patton and Timmermann (2012) denonstrate that if forecasters are rational and employ a correct model specification, and in the absence of measurement error, then MSFE declines with the forecast horizon. In the nowcasting context, this would imply that MSFE decreases when more information is added and the nowcast horizon shrinks up until the publication date of the target variable. By providing a test for nowcast monotonicity the results may thus be interpreted as a check for correct nowcast model specification. Alternatively, if the test is used to evaluate externally-made predictions taken from institutional nowcasters, the test can take on the interpretation as a test for 'nowcaster rationality'. 


\section{ACCEPTED MANUSCRIPT}

In this paper, we provide a formal and robust test for nowcast monotonicity based on the many moment inequalities procedure of Chernozhukov et al. (2014). Our test is the first rigorous procedure to assess nowcast performance, and can be used in very general settings. This improves upon the majority of the aforementioned empirical studies, such as Marcellino et al. (2016), which simply use graphical evidence of declining nowcast MSFE and do not formally test for monotonicity. It also improves on some existing papers that do test for monotonicity, such as Bańbura et al. (2013) who apply the test of Patton and Timmermann (2012), which are only set up to deal with a small nuimber of moment inequalities. Our paper, instead, allows for high-dimensional sets of moment inequalìies driven by the recent interest in 'big data' nowcasting methods.

Relative to the existing econometric literature of forecast evaluation testirg, opin paper provides several new contributions: our first and main contribution is that we alløw for the possibility that the number of nowcasts per quarter, $S$, grows to infinity $(S \rightarrow \infty)$ and therefore so does the number of moment inequalities. That is, while our method is still applicable in finite-dimensional nowcast model approaches such as bridge equations (Schumacher, 2016) or MIDAS models (Clements and Galvão, 2008, 2009) where the number of moment comparisons is typically small (relative to the sample size), it additionally accommodates big data approaches such as the use of factor models which have become widely used for nowcasting (Bańbura et al., 2513, Foreni and Marcellino, 2014, Bańbura and Modugno, 2014 and others). Specifically, we extend the many moment inequalities framework of Chernozhukov et al. (2014), originally geared towards microeconomic applications, to the case of nowcasting. In doing so, we focus on the case ff lartor-based nowcasting methods as the issue of factor estimation in nowcast evaluation is of separate econometric interest.

A second contribution of the paper is that we consider the issue of parameter as well as factor estimation error. We deal with parameter estimation error resulting from the use of the pseudo out-ofsample appraa of West (1996), and derive rate conditions under which it does not affect the validity of critical-values from the high-dimensional moment inequality testing procedure of Chernozhukov et 21.2011 . Niore specifically, we find that, in allowing for $S \rightarrow \infty$, there is a cost to pay through a stronge ate condition relative to papers such as White (2000). Likewise, we provide the conditions reguired for using factor-augmented models of Stock and Watson (2002a b) and Bai and Ng (2006) in this nowcast monotonicity test, with the factors estimated by Principal Components Analysis (PCA), after solving the issue of the 'ragged edge'. This is important as existing papers on the estimation of dynamic factor models with missing observations, such as the Kalman filtering approach of Doz et al. (2011), have focused on the properties of the factor estimates themselves and give no guidance 


\section{ACCEPTED MANUSCRIPT}

in our nowcast evaluation context. In a similar way to the case of parameter estimation error, we find that the rates of Bai and $\mathrm{Ng}(2006)$ have to be tightened in order for factor estimation error not to contribute asymptotically to the critical values of the test.

Finally, we investigate the performance of our test in finite samples through an extensive range of Monte Carlo simulations. These allow for non-monotonicity to occur from different forms of model misspecification and investigate sensitivity to parameter and factor estimation error, as well as varying the number of moment inequalities and out-of-sample splits. Our findings suggest that our tesi performs well across different scenarios, even in the presence of estimation error. We then apply eur test to nowcasting the aggregate GDP growth rate in the United States, as well as five GDP subcemponents, using a data specification similar to that of Bańbura and Modugno (2014). As a preview of the results, our test confirms that there is no statistical evidence of non-monotonicity in the aggregate GDP growth rate. This is in line with previous studies such as Bańbuira et al. (2013), which use a different factor estimation procedure and time period, and seems to indicate that the finding of monotonicity is robust. On the other hand, in the government spending sub-component of GDP our test finds significant violations of monotonicity. This suggests that a different model should be employed for nowcasting government spending. The resulis are very robust to different data spans, dataset configurations and estimation schemes.

The rest of the paper is organised as follows. Section 2 describes the nowcast monotonicity set-up of the paper, and provides details of the factor-augmented model set-up we consider in the theoretical results. Section 3 contains an introduction to the the test statistic, defines the bootstrap critical values and states their formal vahdy. Sestion 4 provides the Monte Carlo simulation settings and results. Section 5 gives the empirical application of the test. Finally, Section 6 concludes the paper. There is also a separate doevment of Supplementary Material which details the proofs of the results of the paper and prordes additional Monte Carlo and empirical results not presented in the main text.

\section{Set-up}

\subsection{Testing Nowcast Monotonicity}

The objective is to evaluate nowcasts of a low-frequency target variable, $y_{t}$, with $T$ observations at quarterly periods $t=1, \ldots, T$. Since data for $y_{t}$ is not published until after the end of quarter $t$, we make a set of $S$ nowcasts at intervals $i=1, \ldots, S$, starting at the beginning of quarter $t$ and ending when $y_{t}$ is published. This is achieved using a large set of $N$ candidate predictors $X_{j t}$, for $j=1, \ldots, N$, 


\section{ACCEPTED MANUSCRIPT}

which are observed at a higher frequency than $y_{t}$. As an example, $X_{j t}$ are taken to be monthly predictors which are observed at $3 T$ monthly periods $t=1 / 3,2 / 3,1, \ldots, T-1 / 3, T$. The extension to higher frequencies is straightforward.

The $S$ different nowcasting horizons are brought about by tracking the arrival of new information in real-time, or the 'data flow' as termed by Bańbura et al. (2013). Assuming that the observations $X_{j t}$ are released sequentially in the order of their $j$ variable index and then in the order of their $t$ time index, the timeline of data releases appears as in Figure 1.

Figure 1: Graphical Illustration of the Data Flow

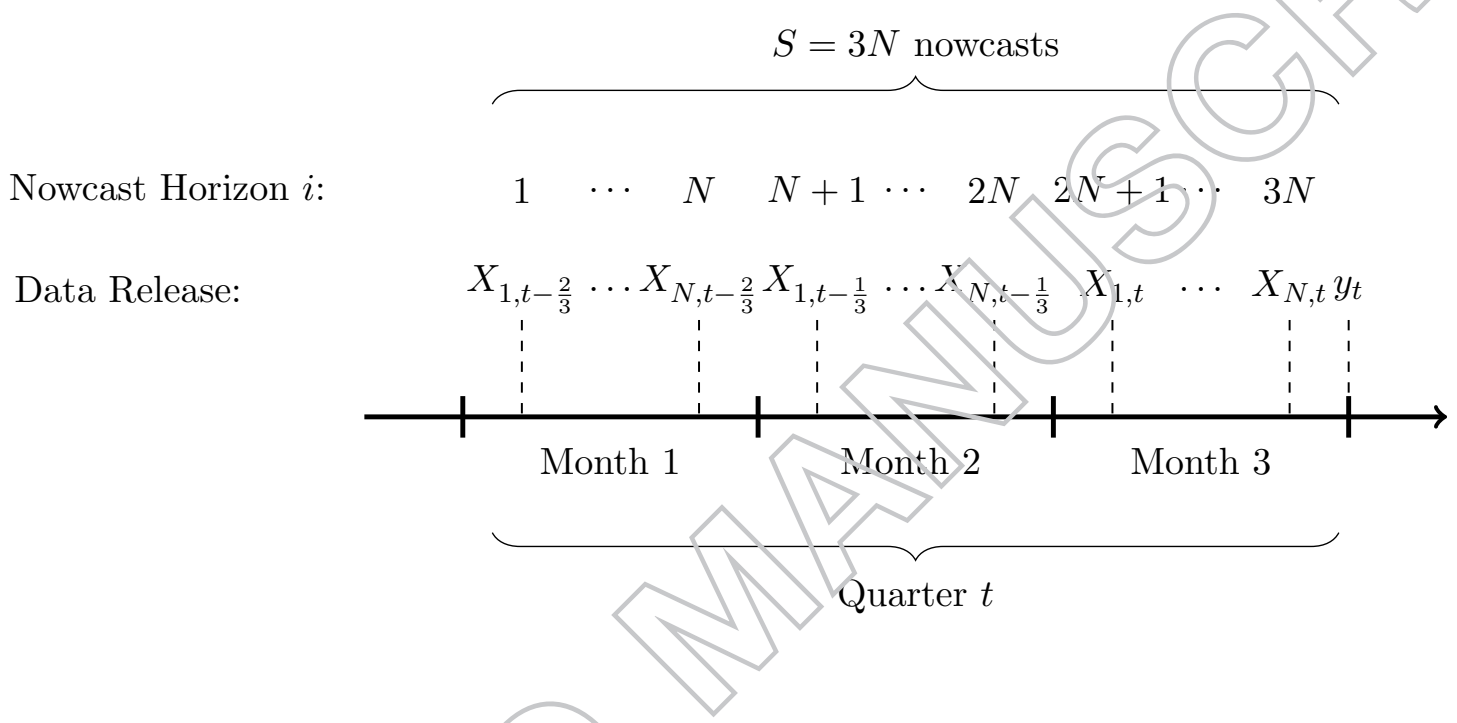

Several comments are useful here. Firstly, this example simplifies the set-up without loss of generality so that $y_{t}$ is released at the end of quarter $t$, whereas in the empirical application we see, for instance, that U.S. GDP data ior ouarter $t$ are, in practice, released some way into quarter $t+1$. Secondly, as we move from $i=1$ through $i=S$, the nowcast horizon shrinks as we approach the fixed publication date of $y_{t}$. This is a slightly different set-up to forecasting studies where the forecast origin is held fixed and the horizon is increased into the future. Finally, it can be seen that the so-called "ragged edge" occurs in the middle of this timeline, where the data are available for some months of the first few variables but are not available for the remainder.

In the above example, the monthly set-up gives rise to a total number of $S=3 N$ different nowcasts per quarter. This number of nowcasts can be made arbitrarily large by increasing the number of variables, $N$. This is particularly relevant for recent "big data" approaches to nowcasting which often allow $N \rightarrow \infty$. The value of $S$ can also be very large when daily predictors are used, or even higher frequency financial series. On the other hand, $S$ could be relatively small if the nowcaster chooses to use one monthly predictor and make $S=3$ nowcasts per quarter. Additionally, some empirical 


\section{ACCEPTED MANUSCRIPT}

nowcasting papers choose a small number of cut-off dates (such as the end of each month) and make only $S=3$ nowcasts based on models which aggregate all of the available data for the previous month. In this paper, we wish to allow for any of these scenarios, and will therefore allow a framework where $S \rightarrow \infty$.

We define $\Omega_{t-1+i / S}$ for $i=1, \ldots, S$ as the collection of data releases at a point $i$ during quarter $t$, which culminates with $\Omega_{t}$ at the end of quarter $t$ where $i=S$ and all of the quarter's data noints have been released. This information set corresponds to all data releases on the left of a given point in the timeline in Figure 1. Then at each point $i$ the nowcaster makes a nowcast based sin the available data at point $i$ and an $\left(m_{i} \times 1\right)$ vector of parameters $\theta_{i}$. We denote this nowcast, which is astally the conditional mean $\mathrm{E}\left[y_{t} \mid \Omega_{t-1+i / S} ; \theta_{i}\right]$, by $y_{i, t}\left(\theta_{i}\right)$ in the following.

Given the motivation in the introduction, the central hypothesis of interest is whether nowcast performance is monotonically improving as we move through the quarter, approaching the publication date of the target variable. Given some loss function $L($.$) , whose properties will be discussed in Section$ 3.2 below, we are interested in knowing whether the nowcast error loss at a point $i+k$ is lower than some earlier point $i$. The null hypothesis is formed of $S(S-1), 2$ moment inequalities for each pairwise comparison of nowcast points $i+k$ and $i$ :

$$
H_{0}: \mathrm{E}\left[L\left(y_{t}-y_{i+k, t}\left(\theta_{i+k}\right)\right)-L\left(y_{t}-y_{i, t}\left(\theta_{i}\right)\right)\right] \leq \mathrm{e} \text { for all } i=1, \ldots, S-1 ; \quad k=1, \ldots, S-i
$$

versus:

$$
H_{1}: \mathrm{E}\left[L\left(y_{t}-y_{i+k, t}\left(\theta_{i+k}\right)-\mathrm{L}\left(y_{t}-y_{i, t}\left(\theta_{i}\right)\right)\right]>0 \quad \text { for some } i=1, \ldots, S-1 ; \quad k=1, \ldots, S-i\right.
$$

The null hypothesis is rolated when at least one point later in the prediction period has larger loss than some earlicr horizon.

Relative to papers such as Patton and Timmermann (2012) for testing forecast monotonicity, we consider ali possible $S(S-1) / 2$ pairwise moment inequalities for the test, rather than just adjacent inequalities, in order to detect any violation of $H_{0}$. Note that, by doing so, even in empirical applicatiens where the number of nowcast points is relatively small, $S(S-1) / 2$ can be very large which further motivates a high-dimensional set-up. We leave the functions $L($.$) and y_{i, t}\left(\theta_{i}\right)$ to be very general in order to incorporate the vast majority of commonly used nowcasting methods, though it is very typical that the MSFE loss function $L(x)=x^{2}$ is used for nowcast evaluation. For $y_{i, t}\left(\theta_{i}\right)$, as mentioned in the introduction, we can easily incorporate different methods operating with a 'small' 


\section{ACCEPTED MANUSCRIPT}

or a 'large' number of regressors.

Since the population parameters $\theta_{i+k}$ and $\theta_{i}$ are unknown, we obtain estimates for the set of moment conditions in $H_{0}$ using a pseudo out-of-sample nowcasting experiment as in West (1996). We split the sample of time series observations, $T$, into samples of size $R$ and $P$. At each of the $P$ quarterly out-of-sample periods $t=R+1, \ldots, T$, we make $i=1, \ldots, S$ different nowcasts of $y_{t}$. We start out estimating the parameters with the first $R$ observations, and then for each $t$ and $i$, we nse ar expanding window of observations using the information set $\left\{\Omega_{s-1+i / S}\right\}_{s=1}^{t}$, known as the recursive scheme. We focus on the recursive rather than the rolling scheme (where the estimatien windioy is fixed at length $R$ ), though the extension of our theoretical results to the latter is straightforward. The focus on the recursive scheme is partly as it is widely used in empirical studies and partiy as we use factor models which are known to be quite robust to structural instabilities, with the latter being one of the main reasons for using the rolling scheme (Clark and McCracken, 2009.

We take the ragged edge at point $i$ into account, and use oniy the information available at point $i$ to estimate $\widehat{\theta}_{i t}$. Therefore, in comparing horizons $i$ and $i+n$, we use sequences of parameter estimates $\left\{\widehat{\theta}_{i t}\right\}_{t=R+1}^{T}$ and $\left\{\widehat{\theta}_{i+k, t}\right\}_{t=R+1}^{T}$. This gives rise to a sample analogute of the moment conditions considered in $H_{0}$ :

$$
\frac{1}{P} \sum_{t=R+1}^{T}\left[L\left(y_{t}-y_{i+k, t}\left(\widehat{\theta}_{i+k, t}\right)\right)-L\left(y_{t}-y_{i, t}\left(\theta_{i t}\right)\right] \quad \text { for } i=1, \ldots, S-1 ; \quad k=1, \ldots, S-i,\right.
$$

which we will use in Section 3 below for our test statistic.

\subsection{Nowcasting with Factor. Augmented Models}

In forming the nowcast oss differentials defined above, of particular interest is when we make nowcasts of the target variable $3^{+}$using the factor augmented model of Stock and Watson (2002a b) and Bai and $\mathrm{Ng}(2006)$, adapter to the case of nowcasting. The simplest version is the unrestricted factor-MIDAS model used by Kim and Swanson (2017) amongst others:

$$
y_{t}=\gamma^{\prime} W_{t}+\beta_{0}^{\prime} F_{t}+\beta_{1}^{\prime} F_{t-1 / 3}+\beta_{2}^{\prime} F_{t-2 / 3}+\varepsilon_{t} \quad t=1,2, \ldots, T
$$

This is a quarterly regression model of $y_{t}$ on $W_{t}$, which is is a set of 'must-have regressors' such as a constant and lags of $y_{t}$, and monthly lags of $F_{t}$, the $r$ unobserved factors based on the monthly static factor model:

$$
X_{t}=\Lambda F_{t}+u_{t} \quad t=1 / 3,2 / 3,1, \ldots, T-2 / 3, T-1 / 3, T
$$




\section{ACCEPTED MANUSCRIPT}

where $X_{t}$ is an $N \times 1$ vector of observed variables, $\Lambda$ is an $N \times r$ matrix of factor loadings, $F_{t}$ is an $r \times 1$ vector of unknown factors and $u_{t}$ is an $N \times 1$ vector of idiosyncratic disturbances. Note that for the theoretical results of the paper we treat the number of factors, $r$, as known whereas in practice this can be consistently estimated by the information criteria of Bai and $\mathrm{Ng}(2002)$ and others. We also abstract from structural breaks in these models, as is common in the forecast evaluation literature. In the empirical application we explore the results using data spans guided by factor loading hreak dates, which can be determined using methods such as Breitung and Eickmeier (2011) and Corradi and Swanson (2014).

We make out-of-sample nowcasts for quarters $t=R+1, \ldots, T$ and nowcast points $l=1 . \ldots, S$ by repeatedly estimating the factors by PCA in expanding monthly windows from observation $1 / 3$ until the end of the quarter $t$, at the various nowcast points $i$. This proceeds in three stages.

In the first stage, we take account of the "ragged-edge" which aceurs at the end of the recursive window estimation sample. Specifically, at points $t=R+1, \ldots . T$ he use $23 t \times N$ window of monthly observations in the matrix $X^{(t)}=\left[X_{1 / 3}, X_{2 / 3}, \ldots, X_{t}\right]^{\prime}$, where the ragged edge only occurs in the last 3 monthly observations of each window. For example if $i \leq N$, then only information is available for month 1 of quarter $t$ up to variable $i$, and no other information is available for the rest of the quarter. In that case, the panel is unbalanced and we cannot directly apply PCA to estimate the factors. We therefore make predictions of the missing observations up to month $t$ and variable $N$ which gives a recursive data matrix:

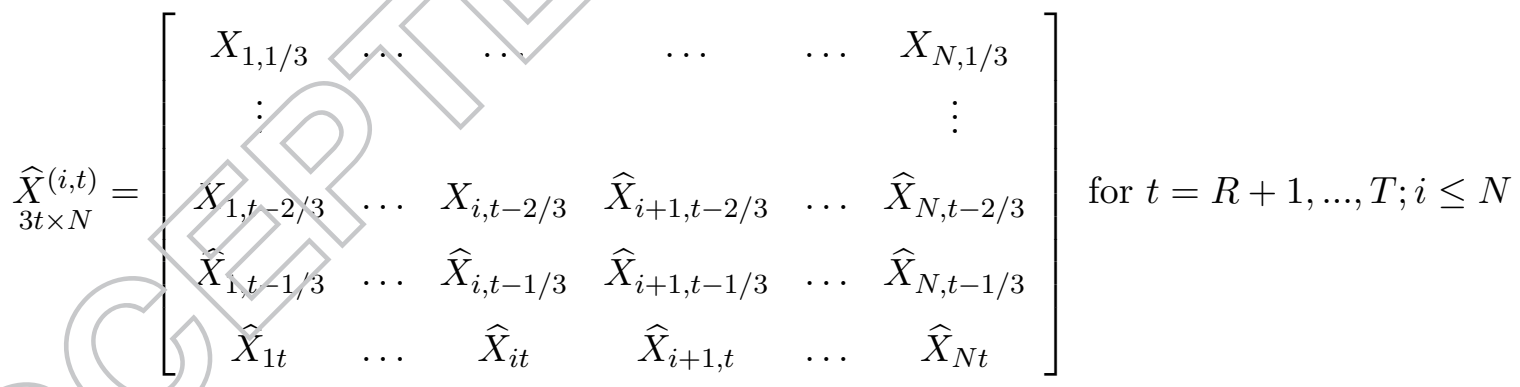

vith typ:cal element $\left\{\widehat{X}_{j}^{(i, t)}: t=R+1, \ldots, T ; j=1 / 3,2 / 3,1, \ldots, t-2 / 3, t-1 / 3, t ; i=1, \ldots, S\right\}$. Analogously, when $N<i \leq 2 N$, the predicted observations only appear in the second-to-last and last row of $\widehat{X}^{(i, t)}$, and when $i>2 N$ they only appear in the last row. This data matrix differs from the out-of-sample factor estimation approaches of Gonçalves et al. (2017) and Fosten (2016) due to these predicted observations in the last three rows to solve the ragged edge. There have been different suggestions of how to balance the panel in the literature. Stock and Watson (2002b) suggest to use an 


\section{ACCEPTED MANUSCRIPT}

EM algorithm to fill in missing observations. A state space approach was suggested by Bańbura and Modugno (2014) using the Kalman filtering method of Doz et al. (2011). Other alternatives include using interpolations to predict missing observations before using PCA (Kim and Swanson, 2017) and vertical alignment (Altissimo et al. 2010).

In the second stage, we estimate the factors by PCA on the data matrix in Equation (5). For) every out-of-sample window $t=R+1, \ldots, T$ and every nowcast point $i=1, \ldots, S$ the PCA optimisation problem is:

$$
\left(\widehat{F}_{3 t \times r}^{(i, t)}, \widehat{\Lambda}_{N \times r}^{(i, t)}\right)=\arg \min _{\Lambda, F}\left\{\frac{1}{3 t N} \operatorname{tr}\left(\widehat{X}^{(i, t)}-F^{(t)} \Lambda^{\prime}\right)\left(\widehat{X}^{(i, t)}-F^{(t)} \Lambda^{\prime}\right\}\right.
$$

subject to the normalizations that $F^{(t) \prime} F^{(t)} / 3 t=I_{r}$ for all $t$ and that $\Lambda^{\prime} \Lambda / N$ is diagonal, where $F^{(t)}=\left[F_{1 / 3}, \ldots, F_{t}\right]^{\prime}$ is a $3 t \times r$ recursive matrix of monthly factors in each quarter $t$, in an analogous way to $X^{(t)}$. The solution is to set $\widehat{F}^{(i, t)}$ as the $r$ eigenvectors corresponding to the $r$ largest eigenvalues of the $3 t \times 3 t$ covariance matrix $\widehat{X}^{(i, t)} \widehat{X}^{(i, t) \prime} / 3 t N$. These eigenvalues are denoted by the $r \times r$ diagonal matrix $\widehat{V}^{(i, t)}$ for each $i$ and $t$. Then since $\widehat{F}^{(i, t) \prime} \widehat{F}^{(i, t)} / 3 t=I_{r}$ (under the normalisation for the factors, we obtain a simple expression for the estimated loadings, $\hat{\Lambda}^{(i, t)}=\widehat{X}^{(i, t) \prime} \widehat{F}^{(i, t)} / 3 t$. The difference to existing papers on factor estimation is the additiond dependence of the estimated factors and loadings on $i$, the nowcast point.

The final stage is to make the noweast. For this we obtain OLS estimates using recursive regressions of $y_{t}$ onto $W_{t}$ and the estimated ractors $\left\{\widehat{F}_{j}^{(i, t)}: t=R+1, \ldots, T ; j=1 / 3,2 / 3, \ldots, t ; i=1, \ldots, S\right\}$. For unrestricted factor-MIDAS model as in Equation (3), the estimated factors are skip-sampled from the monthly frequency and inserter into the regression in a quarterly fashion. In other words, $\widehat{F}_{t}^{(i, t)}$ contains periods $i=-1,2, \ldots, t$, while $\widehat{F}_{t-1 / 3}^{(i, t)}$ contains observations on the second month of every quarter (periods $j=2 / 3,5 / 3, \ldots, t-1 / 3$ ) and $\widehat{F}_{t-2 / 3}^{(i, t)}$ on the first month (periods $j=1 / 3,4 / 3, \ldots, t-$ 2/3). Then, coliecting $\widehat{\boldsymbol{F}}_{t}^{(i, t)}=\left(\widehat{F}_{t}^{(i, t)}, \widehat{F}_{t-1 / 3}^{(i, t)}, \widehat{F}_{t-2 / 3}^{(i, t)}\right)^{\prime}$ and $\widehat{\boldsymbol{\beta}}_{i t}=\left(\widehat{\beta}_{0 i t}^{\prime}, \widehat{\beta}_{1 i t}^{\prime}, \widehat{\beta}_{2 i t}^{\prime}\right)^{\prime}$, the nowcasts are written:

$$
y_{i, t}\left(\widehat{\boldsymbol{F}}_{t}^{(i, t)}, \widehat{\boldsymbol{\beta}}_{i t}, \widehat{\gamma}_{i t}\right)=\widehat{\gamma}_{i t}^{\prime} W_{t}+\widehat{\beta}_{0 i t}^{\prime} \widehat{F}_{t}^{(i, t)}+\widehat{\beta}_{1 i t}^{\prime} \widehat{F}_{t-1 / 3}^{(i, t)}+\widehat{\beta}_{2 i t}^{\prime} \widehat{F}_{t-2 / 3}^{(i, t)}
$$

tor $t=R+1, \ldots, T$ and $i=1, \ldots, S$. These nowcasts are then used directly in Equation (2) to calculate the loss differential series.

Note that, in the absence of measurement error, if we have correctly specified the model for the data generating process, we expect the null hypothesis of monotonicity to hold. Heuristically this is so because, as $i$ grows towards $S$ and we add more and more information, the predictions used to 


\section{ACCEPTED MANUSCRIPT}

solve the ragged edge in $\widehat{X}^{(i, t)}$ are increasingly replaced with actual observations. This implies that the effect of the factors being contaminated with ragged edge predictions vanishes throughout the prediction period, meaning that measures like MSFE are expected to decline as we move towards the end of the nowcast period.

\section{$3 \quad$ Test Statistic and Critical Values}

\subsection{Test Statistic}

To test the null hypothesis $H_{0}$ in Equation (1) we use a statistic which is based on the empirical moment inequalities introduced in Equation (2). That is, the test statistic is chosen to be a max statistic of the following form:

$$
U^{*}=\max _{i, k \in \mathcal{C}_{S}} \frac{1}{\sqrt{P}} \sum_{t=R+1}^{T}\left[L\left(y_{t}-y_{i+k, t}\left(\widehat{\theta}_{i+k, t}\right)\right)-L\left(y_{t}-y_{j}, t\left(\widehat{\theta}_{i t}\right)\right)\right],
$$

where the max could be taken over different sets of moment inequaility comparisons, for example the set of all possible $S(S-1) / 2$ pairwise comparisons $\mathcal{C}_{S}=\{i, k: i=1, \ldots, S-1, k=1, \ldots, S-i\}$, or the set of only adjacent comparisons $\mathcal{C}_{S}=\{i, k: i=1, \ldots, S-1, k=i+1\}$. The null hypothesis of monotonicity, $H_{0}$, in Equation (1) is then rejected if $U^{*}>c(\alpha)$ with $c(\alpha)$ denoting a corresponding critical value at significance level $\alpha$. Reiection of the null implies that there is at least one point later in the quarter which has significartly larger nowcast error loss than at some earlier horizon.

Before describing how to construct the critical values, there is a remark to be made with respect to the set of moment inequalities to be used. Note that in order to apply the results of Chernozhukov et al. (2014) formally we need that for every given $i$ and $k$ combination, the variance of $L\left(y_{t}-y_{i+k, t}\left(\theta_{i+k}\right)\right)-$ $L\left(y_{t}-y_{i, t}\left(\theta_{i}\right)\right.$, 1.e. $v_{i+\hbar, i}^{2} \equiv \operatorname{Var}\left(L\left(y_{t}-y_{i+k, t}\left(\theta_{i+k}\right)\right)-L\left(y_{t}-y_{i, t}\left(\theta_{i}\right)\right)\right)$, is bounded away from zero (a consequence of Assumption SM2 in the Supplementary Material). If we use the factor-based approach of the previous section and re-estimate on every data release, the factors estimated using adjacen releases differ only by the new data point on a single variable out of the total set of $N$ variables. Therefore in the limit as $N \rightarrow \infty$, adjacent horizons' factors become perfectly correlated and $L\left(y_{t}-y_{i+1, t}\left(\theta_{i+1}\right)\right)-L\left(y_{t}-y_{i, t}\left(\theta_{i}\right)\right)=0$ almost surely for every $t$, which implies that $v_{i+k, i}^{2}=0$.

If $N$ is very large, a possible solution to this problem is to drop a subset of moment inequalities which are close together, by bounding the release horizons sufficiently far apart by some fraction of $N$. This can be done in such a way that $v_{i+k, i}^{2}$ is not equal to zero. We use this general approach to prevent $v_{i+k, i}^{2}=0$ in comparing $i$ to $i+k$ by setting $k$ to be at least equal to some deterministic 


\section{ACCEPTED MANUSCRIPT}

sequence which we call $k_{S}^{c}$. That is, for some $S>1$, let:

$$
k_{S}^{c}=\min \left\{k \in\{1, \ldots, S-i\}: v_{i+k, i}^{2}>c>0 \quad \forall i=1, \ldots, S-1\right\}
$$

Intuitively, $k_{S}^{c}$ is the smallest integer $k$ for which the variance $v_{i+k, i}^{2}$ is larger than some constant $c$ for any $i$. Note that this still implies that we make use of every data release in the out-of-sample nowcasting experiment, but merely drop some of the pairwise comparisons of these quantities in forming the test statistic. A regularity condition (see below) then rules out the occurrence of pathological cases of non-monotonicity, which may arise by ignoring the horizons between $i+k_{S}^{c}$ and $i$.

Finally, we point out that, while $v_{i+k, i}^{2}=0$ might be a theoretical concern in the limit, our simulation results as well as our empirical application support the view that this spacing is of minor practical relevance in finite sample settings. Indeed, even in the limit, $v_{i+k, t}^{2}=0$ is typically also not an issue at all if the factors are re-estimated only on a fixed number of calenuar dates such as the end of each month, as described above.

\subsection{Critical Values}

Before we introduce the critical values for our test and establish their formal validity, we introduce the shorthands $\Delta L_{t}\left(\theta_{i+k}, \theta_{i}\right)=L\left(y_{t}-y_{i+k, t}\left(\theta_{\imath+\kappa}\right)\right)-L\left(y_{t}-y_{i, t}\left(\theta_{i}\right)\right)$ and $\Delta L_{t}\left(\widehat{\theta}_{i+k, t}, \widehat{\theta}_{i t}\right)=L\left(y_{t}-\right.$ $\left.y_{i+k, t}\left(\widehat{\theta}_{i+k, t}\right)\right)-L\left(y_{t}-y_{i, t}\left(\widehat{\theta}_{i t}\right)\right)$ for any $i=1, \ldots, S-1$ and $k=1, \ldots, S-i$ combination. Moreover, let $\kappa$ denote the cardinality of $\mathcal{C}_{S}$, i.e. $\ll\left|\mathcal{C}_{S}\right|$.

In order to establish critical valyes for the statistic in (8) such that the test has asymptotic size $\alpha \in(0,1)$, we adost the approach to testing many moment inequalities recently introduced by Chernozhukov et 21. (2014). This procedure does not refer to the asymptotic distribution of the test statistic, but elies on finite sample approximations of the (unknown) asymptotic distribution of the test statistic unaer $H_{0}^{\prime}$ through the use of the Block Multiplier Bootstrap (BMB) procedure. That is, Chernozhukov et al. (2014) show that, under certain regularity conditions, the distribution of $\overbrace{\operatorname{rax}_{i, k} \in \mathcal{C}_{S}} \frac{1}{\sqrt{P}} \sum_{t=R+1}^{T}\left(\Delta L_{t}\left(\theta_{i+k}, \theta_{i}\right)-\mathrm{E}\left[\Delta L_{t}\left(\theta_{i+k}, \theta_{i}\right)\right]\right)$, the statistic evaluated at the true $\left(\theta_{i}^{\prime}, \theta_{i+k}^{\prime}\right)^{\prime}$ and re-centered by $\mathrm{E}\left[\Delta L_{t}\left(\theta_{i+k}, \theta_{i}\right)\right]$, can be approximated directly by that of $\max _{1 \leq l \leq \kappa} Y_{l}$ with $Y=$ $\left\langle Y_{1}, \ldots, Y_{\kappa}\right)^{\prime}$, a centered normal random vector of dimension $\kappa$ (recall that $\kappa$ denotes the cardinality of the set $\mathcal{C}_{S}$ ) with covariance matrix $\mathrm{E}\left[Y Y^{\prime}\right]$, in Kolmogorov distance. This approximation holds for a given sample size $T$ (and other fixed parameter values) up to some bound $C^{\prime} P^{-c^{\prime}}$, where $c^{\prime}$ and $C^{\prime}$ are constants which exclusively depend on some unique constants $c_{1}, C_{1}$, and $c_{2}$ (see below for a definition). Since the covariance structure of $\max _{1 \leq l \leq \kappa} Y_{l}$ is unknown, a similar approximation with 


\section{ACCEPTED MANUSCRIPT}

$\max _{1 \leq l \leq \kappa} Y_{l}$ can also be established for the BMB bootstrap statistic described in the following. Our theoretical results in Theorem 1 below then suggest that the actual test statistic, which under $H_{0}$ satisfies:

$$
U^{*}=\max _{i, k \in \mathcal{C}_{S}} \frac{1}{\sqrt{P}} \sum_{t=R+1}^{T} \Delta L_{t}\left(\widehat{\theta}_{i+k, t}, \widehat{\theta}_{i t}\right) \leq \max _{i, k \in \mathcal{C}_{S}} \frac{1}{\sqrt{P}} \sum_{t=R+1}^{T}\left(\Delta L_{t}\left(\widehat{\theta}_{i+k, t}, \widehat{\theta}_{i t}\right)-\mathrm{E}\left[\Delta L_{t}\left(\theta_{i+k}, \theta_{i}\right)\right]\right)
$$

can indeed be approximated by $\max _{i, k \in \mathcal{C}_{S}} \frac{1}{\sqrt{P}} \sum_{t=R+1}^{T}\left(\Delta L_{t}\left(\theta_{i+k}, \theta_{i}\right)-\mathrm{E}\left[\Delta L_{t}\left(\theta_{i+k}, \theta_{i}\right)\right]\right)$. In other words, Theorem 1 below establishes that parameter and factor estimation error in the test as yell as in the bootstrap statistic can be bounded by $C_{1} P^{-c_{2}}$, where the bound holds again for fixed values of $T, \kappa$, and the other parameters. This suggests that both, the approximation erior with the max of a Gaussian process as well as the factor and parameter estimation error, become negligible asymptotically as $P \longrightarrow \infty$.

Paralleling Chernozhukov et al. (2014), we apply the 'small and 'iarge' blocks technique widely attributed to the paper of Bernstein (1927). That is, let $q_{P}>r_{P}$ denote the 'large' and 'small' blocks respectively and assume that $q_{P}+r_{P}<P / 2$. In analogy to their paper, define $I_{1}=\left\{R+1, \ldots, R+q_{P}\right\}$, $J_{1}=\left\{R+q_{P}+1, \ldots, R+q_{P}+r_{P}\right\}, \ldots, I_{m_{P}}=\left\{R+\left(m_{P}-1\right)\left(q P+r_{P}\right)+1, \ldots, R+\left(m_{P}-1\right)\left(q_{P}+r_{P}\right)+q_{P}\right\}$, $J_{m_{P}}=\left\{R+\left(m_{P}-1\right)\left(q_{P}+r_{P}\right)+q_{P}+1, \ldots, R+m_{i}\left(q_{P}+r P\right)\right\}$, and $J_{m_{P}+1}=\left\{R+m_{P}\left(q_{P}+r_{P}\right)+1, \ldots, T\right\}$. Thus $m \equiv m_{P}$ defines the number of blocks as the integer part of $m=P /\left(q_{P}+r_{P}\right)$ and so $\left(q_{P}+r_{P}\right)$ characterizes an independent block

The algorithm of the block multip lier botstrap is now as follows:

1. Generate standard normal rand.om variables $\epsilon_{1}, \ldots, \epsilon_{m}$ independent of the data $\left\{\Delta L_{t}\left(\widehat{\theta}_{i+k, t}, \widehat{\theta}_{i t}\right)\right\}_{t=R+1}^{T}$.

2. Retaining oniy the large blocks $I_{h}, h=1, \ldots, m_{P}$, construct the BMB statistic:

$$
W_{B M B}=\max _{i, k \in \mathcal{C}_{S}}\left(\frac{1}{\sqrt{m_{P} q_{P}}} \sum_{h=1}^{m_{P}} \epsilon_{h} \sum_{j \in I_{h}}\left(\Delta L_{j}\left(\widehat{\theta}_{i+k, j}, \widehat{\theta}_{i, j}\right)-\frac{1}{P} \sum_{t=R+1}^{T} \Delta L_{t}\left(\widehat{\theta}_{i+k, t}, \widehat{\theta}_{i, t}\right)\right) .\right.
$$

3. Calculate $c^{B M B}(\alpha)$ as the conditional $(1-\alpha)$ quantile of $W_{B M B}$ given $\left\{\Delta L_{t}\left(\widehat{\theta}_{i+k, t}, \widehat{\theta}_{i t}\right)\right\}_{t=R+1}^{T}$. The above algorithm has been designed for a fixed large block-small block combination, and size as well as power of the test may be sensitive to the actual choice. As standard selection methods of the block size for dependent data such as Bühlmann and Künsch (1999) or Hall et al. (1995) have not yet been extended to high-dimensional data, in the Supplementary Material we propose an ad-hoc nested bootstrap procedure suggested by Zhang and Cheng (2014) to estimate the optimal small-large 


\section{ACCEPTED MANUSCRIPT}

block combination. Without claiming optimality of any kind, this choice can then subsequently be employed to perform the moment inequality test of interest on the original sample. The proposed method appears to perform well in Monte Carlo and empirical settings.

For the regularity conditions outlined below, let $c, c^{\prime}, C$, and $C^{\prime}$ denote generic positive constants that may vary throughout the rest of the paper and that are assumed to depend exclusively on $0<c_{1} \leq C_{1}<\infty$ and on $0<c_{2}<1 / 4$. Also, define the $k$-th mixing coefficient for $\beta$-mixing data $\left\{\Delta L_{t}(\cdot)\right\}_{t=1}^{T}$ as:

$b_{\tau}=b_{\tau}\left(\left\{\Delta L_{t}(\cdot)\right\}_{t=1}^{T}\right)=\max _{1 \leq d \leq T-k} \beta\left(\sigma\left(\Delta L_{1}(\cdot), \ldots, \Delta L_{d}(\cdot)\right), \sigma\left(\Delta L_{d+\tau}(\cdot), \ldots, \Delta L_{T}(\cdot), \quad 1 \leq \tau \leq T-1\right.\right.$, where $\sigma\left(\Delta L_{t}(\cdot), t \in \mathcal{T}\right)$ with $\mathcal{T} \subset\{1, \ldots, T\}$ is the sigma field generated by $\Delta L_{t}(\cdot), t \in \mathcal{T}$. We impose the following regularity conditions:

Assumption 1. For every $i, k \in \mathcal{C}_{S}$, the data $\left\{\Delta L_{t}\left(\theta_{i+k}, \theta_{i}\right)\right\}_{+=1}^{T}$ is strictly stationary (across $t$ ) and $\beta$-mixing where the size of the $\tau-t h$ mixing coefficient is determinea in Assumption 4 below.

Assumption 2. Assume that:

$$
E\left[\max _{i, k \in \mathcal{C}_{S}}\left|\Delta L_{t}\left(\theta_{i+k}, \theta_{i}\right)-E\left[\Delta L_{t+1}\left(\theta_{i+k}, \theta_{i}\right)\right]\right|^{s}\right]<C_{1} \quad 1 \leq s \leq 4 .
$$

as well as:

$$
E\left[\| \nabla_{F} \Delta L_{t}\left(\rho_{i+k}, \theta_{i} j \|^{4 d_{r_{F}}}\right]<C \quad \text { and } E\left[\left\|\nabla_{\beta} \Delta L_{t}\left(\theta_{i+k}, \theta_{i}\right)\right\|^{4 d_{r_{P}}}\right]<C\right.
$$

for all $t=1, \ldots, T$ ana $i, k \in \mathcal{C}_{S}$, where $d_{r_{P}}$ is again defined in Assumption 4 below.

Assumption 3. For every $t=1, \ldots, T$, and $i, k \in \mathcal{C}_{S}$, in an open neighbourhood of $\theta_{i+k}$ and $\theta_{i}$, the function $\Delta D_{t}\left(\theta_{\imath+k}, \theta_{i}\right)$ is measurable and continuously differentiable with respect to all elements of $\theta_{i}$ and $\theta_{i+k}$ with probability one.

Assumption 4. Let $R, P, N \longrightarrow \infty$ as $T \longrightarrow \infty$ and assume that there exists a sequence $\zeta_{P 1} \longrightarrow 0$ as $P \longrightarrow \infty$. Recalling $c_{2}$ defined above, we impose the following conditions: let $P / R^{\frac{1}{2}} \longrightarrow \infty$ and $P / N=O(1)$, and assume that

$$
C^{\prime} \max \left\{\frac{P^{1+c_{2}}}{R}, \frac{P^{\frac{1}{2}+c_{2}}}{N}\right\} \leq \zeta_{p 1} \leq \frac{C_{1}}{P^{c_{2}}}
$$

Moreover, assume that the block sizes satisfy $q_{P}=o(P), r_{P}=o\left(q_{P}\right)$ as $P \longrightarrow \infty$. The mix- 


\section{ACCEPTED MANUSCRIPT}

ing coefficient $b_{r_{P}}$ and the block sizes $r_{P}$ and $q_{P}$ together with the number of blocks $m_{P}$ satisfy $\max \left\{m_{P} b_{r_{P}},\left(r_{P} / q_{P}\right) \log ^{2} \kappa\right\} \leq C_{1} P^{-c_{2}}$ and $q_{P} C \log ^{\frac{5}{2}}(\kappa P) \leq C_{1} P^{\frac{1}{2}-c_{2}}$, where $b_{r_{P}}=o\left(r_{P}^{-2 d_{r_{P}} /\left(d_{r_{P}-1}\right)}\right)$ and $d_{r_{P}}>1$.

Assumption 5. For some c defined before, the sequence $k_{S}^{c_{1}}$ defined in (9) exists for every $S=1,2, \ldots$ and for every $i$,

$$
\operatorname{Pr}\left(\left\{\max _{1 \leq t \leq T, k<k_{S}^{c_{1}}} \Delta L_{t}\left(\theta_{i+k}, \theta_{i}\right)>0\right\} \cap\left\{\max _{1 \leq t \leq T, k \geq k_{S}^{c_{1}}} \Delta L_{t}\left(\theta_{i+k}, \theta_{i}\right) \leq 0\right\}\right) \rightarrow 0
$$

as $S, T \longrightarrow \infty$.

Assumption 1 characterizes the time dependence in the data, while Assumption 2 requires the existence and finiteness of certain moment expressions. Note that the mornent conditions in Assumption 2 involve the loss function, and thus the set of admissible nowcast errer distributions which satisfy Assumption 2 will in fact depend on the actual loss function empipyed. We point out, however, that when nowcast errors are for instance normally distributed with firite mean and variance for every $i=1, \ldots, S$, then the moment conditions in the first pait of Assumption 2 are satisfied for commonlyused loss functions such as the symmetric squared error loss or the asymmetric linear exponential (Linex) loss. Assumption 3 on the other hand requires differentiability of $\Delta L_{t}\left(\theta_{i+k}, \theta_{i}\right)$, which rules out loss functions such as mean absolüte deviation loss, but could, in principle, be weakened along the lines of McCracken (2000). Assumption 4 modifies the asymptotics as in West (1996) with new rates due to our nowcasting se-up. By using big-data approaches allowing the number of moment inequalities, $\kappa=\kappa(N)$, to grow to jifinity with the number of variables $N$ and with the sample size split into $T=R+P$ this assumption governs the interplay of these three sequences. Also noting that with calendar release dates linked to the number of variables, meaning $\kappa$ will at most be of order $O\left(N^{2}\right)$, the first part of Assumption 4 requires $R$ to grow slower than $P^{2}$, but faster than $P^{1+2 c_{2}}$, where $0<c_{2}<1 / 4$. This is slightly stricter than $P / R \rightarrow 0$, the requirement imposed by West (1990) to ignore parameter estimation error asymptotically, and, when $c_{2}=\frac{1}{8}$ for instance, allows for $\left.R \in\left(c P^{5}\right), C P^{2}\right)$. Similarly, we allow the number of variables $N$ to grow as fast as $P$, but require that $\left(R^{\frac{1}{2}}+2 c_{2} / N\right) \longrightarrow 0$, a condition which is again slightly stricter than the out-of-sample analogue of Bai and $\mathrm{Ng}$ (2006) who require $\sqrt{P} / N \rightarrow 0$ to eliminate factor estimation error asymptotically. This tightening of the growth rates may be viewed as the 'price to pay' to let the number of moment inequalities grow with the sample size. For instance, if again $c_{2}=\frac{1}{8}$, we can allow for $N \in\left(c P^{\frac{3}{4}}, C P\right)$, which in turn implies that $N / T \longrightarrow 0$. The second part of Assumption 4 determines the rate of the small $\left(r_{P}\right)$ 


\section{ACCEPTED MANUSCRIPT}

and large block $\left(q_{P}\right)$ sizes, respectively, and sets the mixing size to be of order $-2 d_{r_{P}} /\left(d_{r_{P}}-1\right)$. It postulates that the rate of the large block size $q_{P}$ is at most of order $P^{\frac{1}{2}-c_{2}}$ (if $c_{2}=\frac{1}{8}: q_{p}<C P^{\frac{3}{8}}$ ), while $r_{P}$ in this case may only grow up to rate $P^{\frac{1}{2}-2 c_{2}}$ (if $c_{2}=\frac{1}{8}: r_{p}<C P^{\frac{1}{4}}$ ). Finally, note that the mixing condition is slightly weaker than in West (1996) who imposes a mixing coefficient of size $-3 d /(d-1)$ and thus allows for less time dependence in the data. Finally, Assumption 5 rules out that,, as $S$ and $T$ grow to infinity, any violations of monotonicity remain undetected by spacing moment comparisons $k_{S}^{c_{1}}$ periods apart from each other. This ensures that the null hypothesis $H_{2}$ remains unaffected in the limit by using this spacing.

Before we state the main theoretical result, we point out that there are two additional assumptions in the Supplementary Material, Assumptions SM1 and SM2, which are required for the statement in Theorem 1 1 below. More specifically, Assumption SM1 contains rather standard factor model regularity conditions adapted to the recursive parameter estimation case, many of which are similar or even identical to Gonçalves et al. (2017). By contrast, Assumption SM2 is needed to formally relax a bounded support assumption made by Chernozhukov et a! 2014) for the case of dependent data for illustrative purposes. However, these are not presented here a'ue to their long and mainly technical nature. The following Theorem establishes the validity of the bootstrap critical values using the BMB procedure:

Theorem 1. Recall the definition of the constants and assume that Assumptions 1, 2, 3, 4, 5 hold, as well as SM1 and SM2 from the Supplernentary Material. Then, there exist positive constants c, C such that under $H_{0}$ :

$$
\operatorname{Pr}\left(U^{*}>c^{B M B}(\alpha)\right) \leq \alpha+C P^{-c} \text {, }
$$

where $c^{B M B}(\alpha)$ is ihe corresponding critical value at level $\alpha$ from the Block Multiplier Bootstrap procedure descibed bejare. If $E\left[\Delta L_{t}\left(\theta_{i+k}, \theta_{i}\right)\right]=0$ for all $i, k \in \mathcal{C}_{S}$, then:

$$
\left|\operatorname{Pr}\left(U^{*}>c^{B M B}(\alpha)\right)-\alpha\right| \leq C P^{-c} .
$$

Theorem 1 yields critical values for $U^{*}$ in such a way that the test has asymptotic size $\alpha \in(0,1)$.

Note however that the bounds in Theorem 1 are non-asymptotic in the sense that the bound $C P^{-c}$ holds for a fixed value of $P$ and $\kappa$ (and the fixed parameters). The second part of Theorem 1 , namely Equation (11), shows that when all moment inequalities are binding, the asymptotic size of the test coincides with the nominal size $\alpha$. 


\section{ACCEPTED MANUSCRIPT}

\subsection{Critical Values with Moment Inequality Selection}

When the number of non-binding and thus uninformative moment inequalities with $\mathrm{E}\left[\Delta L_{t}\left(\theta_{i+k}, \theta_{i}\right)\right]<$ 0 is large, one-step critical values described in the previous section may become too conservative. A possible solution to this problem is the application of moment selection procedures which do not consider inequalities in the calculation of the critical values that are unlikely to be binding, i.e. in equalities whose estimated counterpart lies below a certain threshold value. This selection proceaure is similar in spirit to the Superior Predictive Ability test of Hansen (2005), which overcomes poreritial conservativeness of White (2000)'s reality check test for comparing a (finite) number of forecast models by ignoring irrelevant models in the construction of the limiting distribution.

For the moment selection, we adopt a two-step method proposed by Chernozhykpv et al. (2014) for the Multiplier Bootstrap to the BMB used in that paper. Let $0<\beta P<\alpha / 2$ be some constant which may depend on the out-of-sample size $P$. Then let $c^{B M B}\left(\beta_{P}\right)$ analcogusly denote the one-step critical values calculated using the procedure above, for size $\beta_{p}$ rather than $\alpha$. Finally denote the moment inequality selection set $\widehat{J}_{B M B}$ as:

$$
\widehat{J}_{B M B}=\left\{i, k \in \mathcal{C}_{S}: \frac{1}{\sqrt{P}} \sum_{t=R+1}^{T} \widehat{L}_{i}\left(\widehat{\theta}_{i+k, t}, \widehat{\theta}_{i, t}\right)>-2 c^{B M B}\left(\beta_{P}\right)\right\} .
$$

Then the two-step critical values with moment inequality selection, which we denote $c^{B M B, 2 S}(\alpha)$, can be calculated using the following procedure:

1. Generate standard normairandon variables $\epsilon_{1}, \ldots, \epsilon_{m}$ independent of the data $\left\{L_{t}\left(\widehat{\theta}_{i+k, t}, \widehat{\theta}_{i, t}\right)\right\}_{t=R+1}^{T}$.

2. Construct the BMIS statistic $W_{\widehat{J}_{B M B}}$ as:

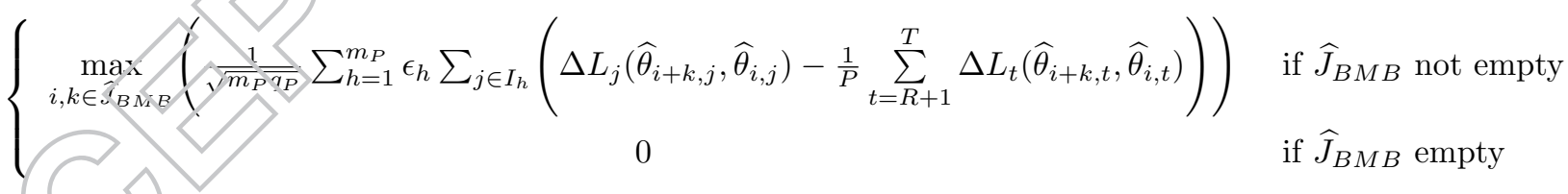

3. Cal cuiate $c^{B M B, 2 S}(\alpha)$ as the conditional $\left(1-\alpha+2 \beta_{P}\right)$ quantile of $W_{\widehat{J}_{B M B}}$ given $\left\{L_{t}\left(\widehat{\theta}_{i+k, t}, \widehat{\theta}_{i, t}\right)\right\}_{t=R+1}^{T}$.

Given this two-step procedure for the computation of critical values, the following result establishes their theoretical validity:

Theorem 2. Recall the definition of the constants and suppose that the assumptions of Theorem 1 hold. Moreover, suppose that $\sup _{P \geq 1} \beta_{P}<\alpha / 2$ and $\log \left(1 / \beta_{P}\right) \leq C_{1} \log P$, where $\beta_{P}$ was defined above. 


\section{ACCEPTED MANUSCRIPT}

Then, there exist positive constants $c$ and $C$ depending only on $c_{1}$ and $C_{1}$ such that under $H_{0}$ :

$$
\operatorname{Pr}\left(U^{*}>c^{B M B, 2 S}(\alpha)\right) \leq \alpha+C P^{-c},
$$

where $c^{B M B, 2 S}(\alpha)$ is the corresponding critical value at level $\alpha$ from the two-step Block Multiplien Bootstrap procedure with moment selection described before. If $E\left[\Delta L_{t}\left(\theta_{i+k}, \theta_{i}\right)\right]=0$ for all $i, k \in \mathcal{C}_{S}$, then:

$$
\operatorname{Pr}\left(U^{*}>c^{B M B, 2 S}(\alpha)\right) \leq \alpha-3 \beta_{P}-C P^{-c},
$$

so that, if in addition $\beta_{P} \leq C_{1} P^{-c_{1}}$, then $\left|\operatorname{Pr}\left(U^{*}>c^{B M B, 2 S}(\alpha)\right)-\alpha\right| \leq C P^{-c}$

Similar to Theorem 1, results are sub-divided into two parts: the first part of Theorem 2 addresses the case where only some inequalities are binding, while the second part consicers the case when all moments are zero, in other words $\mathrm{E}\left[\Delta L_{t}\left(\theta_{i+k}, \theta_{i}\right)\right]=0$ for all $1 \leq \mathrm{V} \leq k$. As expected, the possibility of dropping some inequalities from the set of moment restrictions used for the computation of critical values when they are actually informative, leads to an additional error of order $3 \beta_{P}$. This error, however, becomes negligible when $\beta_{P} \leq C_{1} P^{-c_{1}}$ and the same convergence rate as in Theorem 1 is obtained.

\section{Monte Carlo}

In this section we investigate the finite sample properties of the test statistic in Equation (8). Of particular interest is the performance of the test across different sample sizes, with different specifications of parameter and factoy estimation. We therefore propose two set-ups: the first specifies $y_{t}$ to be a function of a small set of non-factor regressors. This allows us to assess the performance of the test in a lower-dinensional set-up more similar to the MIDAS and bridge equation approaches, and also allorys us to shut down the factor estimation component of the results. The second set-up has both parameter and factor estimation which allows us to investigate the test in high dimensions with factor estimation error. We will check sensitivity to the in-sample and out-of-sample splits, $R$ and $P$ also when the test is constructed using different sets of moment inequalities: the full set of $S(S-1) / 2$ inequalities, the restricted set using spacings of width $k_{S}^{c}$ described in Section 3.1 , and the adjacent-only inequalities version akin to the $\boldsymbol{\Delta}^{\mathbf{e}}$ test of Patton and Timmermann (2012). 


\section{ACCEPTED MANUSCRIPT}

\subsection{Set-up 1: Parameter Estimation Only}

In the first Monte Carlo set-up, $y_{t}$ is a function of a vector of $S$ non-factor variables $Z_{t}=\left[Z_{1 t}, \ldots, Z_{S t}\right]^{\prime}$ :

$$
y_{t}=\gamma^{\prime} Z_{t}+\varepsilon_{t}
$$

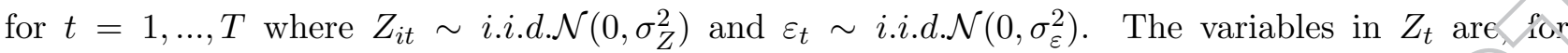
simplicity, taken to be at the same frequency as $y_{t}$ but released at staggered intervals in the order $1, \ldots, S$ in a similar way as depicted in Figure 1. We assume that the nowcaster makes noveasts at each of these points $i=1, \ldots, S$ using a misspecified one-variable model which only uses variable $i$, namely:

$$
y_{t}=\widetilde{\gamma}_{i} Z_{i t}+\varepsilon_{i t} \quad i=1, \ldots, S
$$

The nowcasting model in Equation (15), which omits all but one of the $S$ variables in the true DGP for $y_{t}$, gives a $M S F E_{i}$ at points $i=1, \ldots, S$ of $M S F \mathcal{i}_{i}=\sigma_{\varepsilon}^{2}+\sum_{j \neq i} \gamma_{j}^{2} \sigma_{Z}^{2}$. We can therefore vary the relative magnitudes of the sequence of parameter values $\gamma_{1}, \ldots, \gamma_{S}$ in order to generate different scenarios according to the null and alternative hypotheses.

In order to simulate the least favourable case under the null, where the inequalities in the null hypothesis in Equation (1) all hold with equality, we use the parameterisation:

DGPZ-N: $\gamma_{i}=1$ for all $i=1, \ldots, S$

which gives equal $M S F E_{i}$ for each i. Under the alternative hypothesis we use the parametersations:

DGPZ-A1: $\gamma_{i}=1$ for $i=\lfloor S / 2\rfloor$ and $\gamma_{i}=0$ for $i \neq\lfloor S / 2\rfloor$

DGPZ-A2: $\gamma_{i}=\sqrt{\mathrm{exp}(-i)}$ for all $i=1, \ldots, S$

These give two different types of violations of monotonicity similar to existing studies in the monotonicity testing literature such as Ghosal et al. (2000). Specifically, in DGPZ-A1 there is a oneoft spile in the $\gamma_{i}$ coefficients around the (integer part of) point $S / 2$ which causes a drop down and Immediate jump up in the $M S F E_{i}$ profile. In DGPZ-A2 the $\gamma_{i}$ coefficients decline as $i$ increases, which implies that $M S F E_{i}$ is smoothly increasing across the whole profile.

For the various DGP parameters and sample sizes, we use a number of variables $S \in\{5,10\}$ which allows us to assess the performance of the test in a relatively small-dimensional case. For the allinequalities version of the test this still yields a total of $S(S-1) / 2 \in\{10,45\}$ moment inequalities, 


\section{ACCEPTED MANUSCRIPT}

although we will compare the performance to the adjacent-only version which uses only $S-1 \in\{4,9\}$ inequalities. We let $\sigma_{Z}^{2}=1$ and therefore set $\sigma_{\varepsilon}^{2}=\sum_{i=1}^{S} \gamma_{i}^{2}$ which fixes the signal-to-noise ratio in Equation (14) to be 1:1. The nowcaster uses recursive OLS to estimate the models described in Equation (15). We let the total sample size be $T \in\{200,300\}$ and for the sample split $T=R+P$ we use ratios $P / R \in\{1 / 2,2\}$ to allow cases where $R<P$ and $P>R$, and so $R$ and $P$ vary between 67 and 200 .

For the bootstrap, we considered a grid of values for the small and large blocks equal $\{0,1,2\}$ and $q_{P} \in\{4,5,6,7,8\}$, though we only display some of these combinations for brevity We make use of the warp speed bootstrap of Giacomini et al. (2013) which allows us to make just $B=1$ bootstrap draw over $M=999$ Monte Carlo replications and reduce the computational complexity of the problem.

\subsection{Set-up 2: Parameter and Factor Estimation}

In this next set-up we simulate both the factor model and factor augmented model in Section 2 to give both parameter and factor estimation in the out-of-sample estimation approach. The quarterly factor-augmented regression model is a simplified version of Equation (3):

$$
y_{t}=\beta_{0}^{\prime} F_{t}+\beta_{1}^{\prime} F_{t-1 / 3}+\beta_{2}^{\prime} F_{t-2 / 3}+\varepsilon_{t}
$$

for quarters $t=1,2, \ldots, T$ which has ne additional regressors $W_{t}$. The monthly factor model has $3 T$ observations on the $N \times 1$ vector of variables $X_{t}$ which is exactly as in Equation (4). As above, the variables $X_{j t}$ are released at distrinct times in the order $j=1, \ldots, N$, yielding $S=3 N$ nowcasts per quarter. The set-up is interesting relative to existing simulations such as Gonçalves et al. (2017) which do not allow for the ragged edge or consider multiple updates of factor estimates.

The nowcaster forms nowcasts on each data release through the three months of each quarter. The factors are est mated by recursive PCA after solving the ragged edge using an $A R(1)$ interpolation. The estimated tactors are then used in the second-stage where recursive OLS is used to estimate the nowcast medel. exactly as described in Section 2.2. We take a simple specification of the data generating processes in Equations (16) and (4) where the idiosyncratic errors are $u_{i t} \sim$ i.i.d. $\mathcal{N}\left(0, \sigma_{u}^{2}\right)$, there is a single factor $r=1$ which follows the $A R(1)$ process $F_{t}=\rho_{F} F_{t-1}+v_{t}$ where $v_{t} \sim$ i.i.d.N $\left(0,1-\rho_{F}^{2}\right)$ so that the unconditional variance of the factors is fixed at unity. We let the factor loadings be the non-stochastic vector $\Lambda=\mathbf{1}_{N \times 1}$ and finally the factor-augmented model errors are $\varepsilon_{t} \sim$ i.i.d. $\mathcal{N}\left(0, \sigma_{\varepsilon}^{2}\right)$.

In the results which follow, we do not present simulations under the null. As mentioned in Section 


\section{ACCEPTED MANUSCRIPT}

2.2 , a correct nowcast model specification in this set-up leads to a strictly declining MSFE profile as the contamination to the factors due to the ragged edge interpolation vanishes throughout the period as we add information from $i=1$ to $i=S$. Thus, this downward-sloping MSFE is in the interior of the null space (with $\mathrm{E}\left[\Delta L_{t}().\right]<0$ rather than the least favourable case where $\mathrm{E}\left[\Delta L_{t}().\right]=0$ ) and we therefore expect the rejection rates to be close to zero under this specification. We confirmed this by running results for the case where $\left(\beta_{0}, \beta_{1}, \beta_{2}\right)=(0.25,0.25,0.25)$ which are available from the anf hors on request.

In order to simulate under the alternative, as above we assume the nowcaster repeatedly misspecifies the model for the true DGP in Equation (16). Again, they use a single-variable version of the model which in this case uses only the factor dated in the current month.

$$
\begin{aligned}
& y_{t}=\widetilde{\beta}_{2}^{\prime} F_{t-2 / 3}+\varepsilon_{1 t} \\
& y_{t}=\widetilde{\beta}_{1}^{\prime} F_{t-1 / 3}+\varepsilon_{2 t} \\
& y_{t}=\widetilde{\beta}_{0}^{\prime} F_{t}+\varepsilon_{3 t}
\end{aligned}
$$

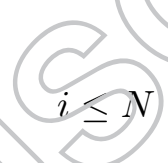

Ignoring the effect of the ragged edge interpolation, the monthly $M S F E_{m}$ for $m=1,2,3$ are equal to $M S F E_{1}=\left(\beta_{0}^{2}+\beta_{1}^{2}\right) \sigma_{F}^{2}+\sigma_{\varepsilon}^{2}, M S F E_{2}=\left(\beta_{0}^{2}+\beta_{2}^{2}\right) \sigma_{F}^{2}+\sigma_{\varepsilon}^{2}$ and $M S F E_{3}=\left(\beta_{1}^{2}+\beta_{2}^{2}\right) \sigma_{F}^{2}+\sigma_{\varepsilon}^{2}$. Therefore monotonicity is violated either when $\beta_{2}>\beta_{1}$ or $\beta_{1}>\beta_{0}$ (or both). We therefore consider two different parameterisations for the alternative with differing magnitudes of non-monotonicity:

DGPF-A1: $\left(\beta_{0}, \beta_{1}, \beta_{2}\right)=(0,0,5,1,5)$

DGPF-A2: $\left(\beta_{0}, \beta_{1}, \beta_{2}\right)=(0,0.5,3)$.

For the varions LGP parameters and sample sizes, we let $\sigma_{u}^{2}=1$ and $\rho_{F}=0.5$ in the factor model. For the nowrast rionel errors we let $\sigma_{\varepsilon}^{2}=\left(\beta_{0}^{2}+\beta_{1}^{2}+\beta_{2}^{2}\right)$ which fixes the signal-to-noise ratio in Equation (16) to be 1:1. The same quarterly sample sizes $T \in\{200,300\}$ are used, noting that this gives monthly sample sizes $3 T \in\{600,900\}$ for $X_{t}$. The same sample splits for $R$ and $P$ are maintained from the previeus section. For the number of variables in $X_{t}$, we consider $N \in\{10,20\}$. This gives a large number of $S \in\{30,60\}$ nowcasts per quarter, which in turn yields a maximum number of moment inequalities of $S(S-1) / 2 \in\{435,1770\}$. Since there may be the issue of highly correlated factor estimates, as outlined in Equation (9) we will check the performance of the all-inequalities version of the test with that of moment inequality spacings of $k_{S}^{c}=5$ (further combinations are considered in the Supplementary Material). 


\section{ACCEPTED MANUSCRIPT}

We note that, although $N=10$ is at the lower bound of the number of series used in empirical factor nowcasting studies, this will allow us to explore the performance of the test under factor estimation error. The simulations of Gonçalves et al. (2017) show that the size and power of their test do not vary significantly with $N$, even if it is as low as 10 . In our case we may expect more variation over $N$ because it implies large differences in the number of inequalities.

\subsection{Results}

Table 1 displays the one-sided rejection rates for the set-up without factor estimation errer (DGPZ-N, DGPZ-A1 and DGPZ-A2) for a nominal size of 5\%. The results are displayed for the bieck choices $r_{P} \in\{0,1,2\}$ and $q_{P} \in\{4,5\}$ and for the all-inequalities and adjacent-only versions of the test. The results show that the test has good size properties for the least favourable case under the null. For the all-inequalities version of the test, rejection rates tend to be close to the rominal size of $5 \%$, in many cases between $4 \%$ and $6 \%$. We see that the test becomes more conservative as the number of inequalities increases, though this is of similar magnitude to the results of Zhang and Cheng (2014), who consider a similar set-up.

In terms of the power, we also see that the test has good performance. For DGPZ-A1, for the all-inequalities version on the left of Table 1 we see power above $90 \%$ everywhere for the $T=200$ case, increasing to unity as $T$ increases to $T=300$. For DGPZ-A2 the power is slightly lower at around $70 \%$ when $P=67$ but this improves significantly with increases in the sample size. However, we find some more worrying ressits when using the adjacent-only inequalities test similar to the $\boldsymbol{\Delta}^{\mathbf{e}}$ test of Patton and Timmermann 2512). Looking at the right hand side of Table 1, although the size properties seem reasonable, we see that the power of this version of the test is substantially lower for DGPZ-A2 which exhibits smoothly upwards-sloping violation of monotonicity.

In general, we conclude that the test works well in this set-up with a small number of non-factor predictors. We also suggest that it is preferable to use the full set of moment inequalities (and not only the adjacent ones), particularly as the results of Theorem 1 allow this to be large relative to the sample size. We now turn our attention to the second case with factor predictors.

In the presence of factor estimates, the results in Table 2 show that the test retains good power properties. The rejection rates are above $80 \%$ in all cases for both DGPF-A1 and DGPF-A2 where the quarterly sample size is $T=200$ and rise to unity when $T=300$. In analysing the effect of parameter and factor estimation error on the results, considering the case where $T=200$ in the top half of Table 2, we are interested in what happens as $(R, P)$ switches from $(133,67)$ to $(67,133)$. We 


\section{ACCEPTED MANUSCRIPT}

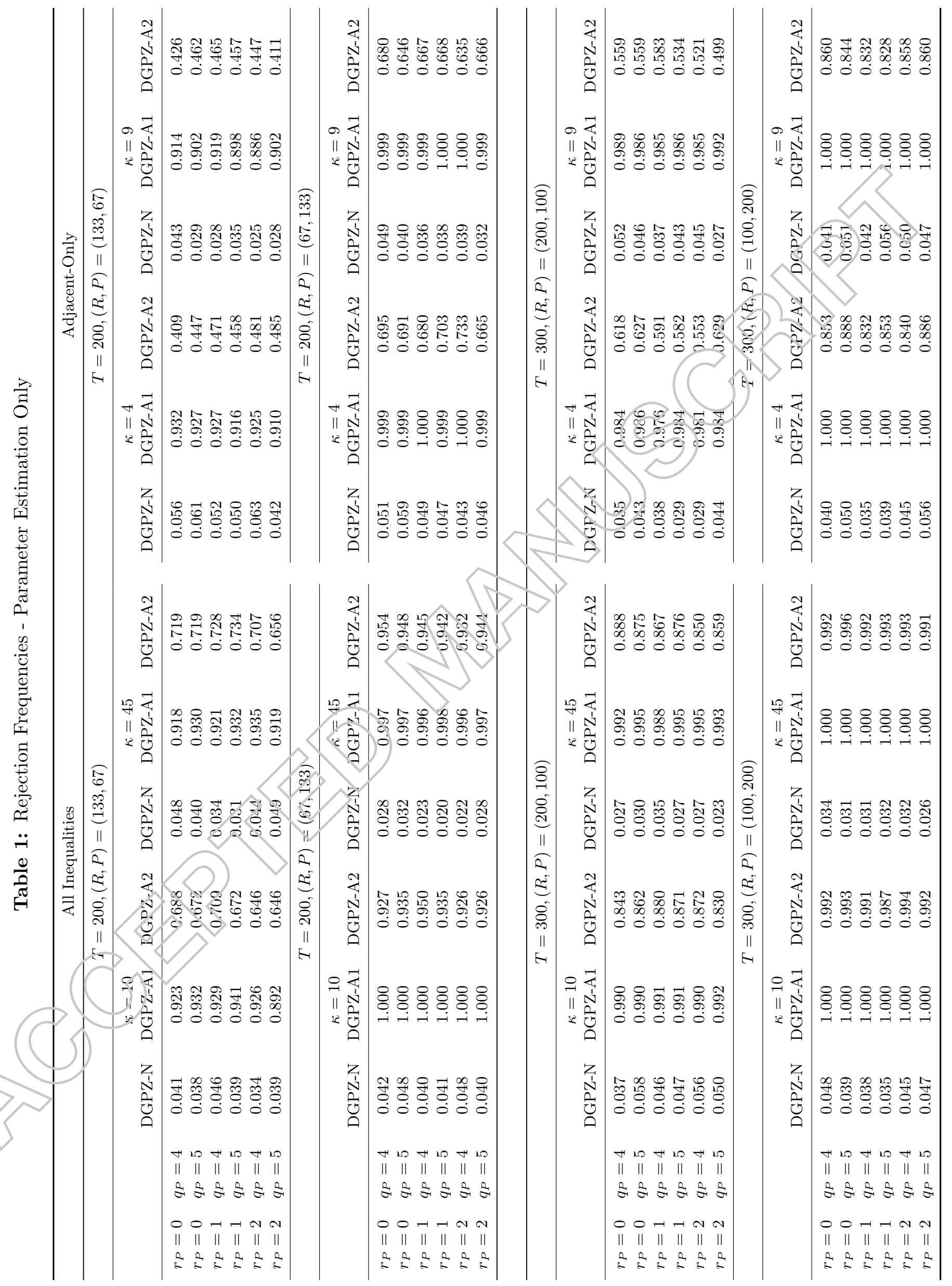




\section{ACCEPTED MANUSCRIPT}

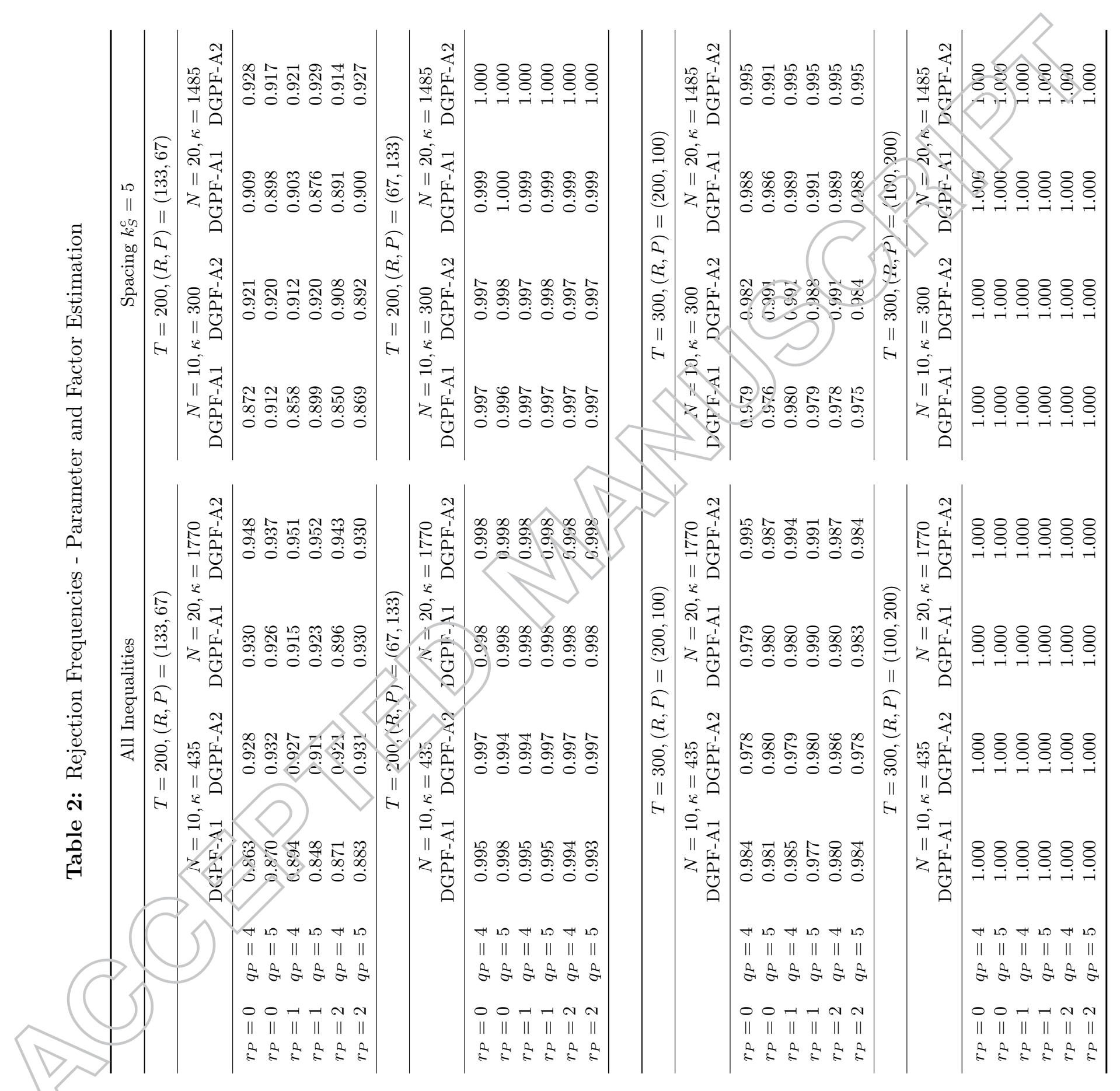




\section{ACCEPTED MANUSCRIPT}

see that the power of the test under DGPF-A1 and DGPF-A2 improves significantly as we move from the $(R, P)=(133,67)$ to the $(R, P)=(67,133)$ case. This indicates that, although $R$ is required to be large relative to $P$ for estimation error to be irrelevant asymptotically, in finite samples it appears that increases in power come from the increase in the number of out-of-sample points, $P$, in spite of larger estimation error.

Table 2 shows that the results do not change markedly when comparing the all-inequalities version of the test to those with spacings $k_{S}^{c}=5$. This indicates that this may not be a serious issue in practical applications. In the Supplementary Material, we provide a wide range of further simulatyons which explore this in more detail, including an alternative set-up where monotonicity is violated when the nowcaster has a horizon-specific prediction bias.

\section{Empirical Application}

In this section we will present the results of our monotonicity test in nowcasting the United States aggregate real GDP growth rate, and the growth rate of five GDP subcomponents. This builds on the literature of nowcasting the individual components of GPP, studies of which are less common than the large volume of empirical studies on nowcasting agregate real GDP. Some examples include Baffigi et al. (2004) who take a bottom-up approach to nowcasting aggregate GDP, and Antolín Díaz et al. (2017) who nowcast real consumption separately to aggregate GDP. We find this to be an important exercise as nowcasting the GDP sub-components to some extent mimics the way that the Bureau of Economic Analysis (BEA constructs the first estimates of aggregate GDP, as mentioned in the Working Paper for the Atlanta Fed's GDPNow model, see Higgins (2014).

\subsection{Descripcijon of Data and Nowcasting Method}

The quarterly real G5P variable and its five sub-components (consumption, investment, government expenditure, exports and imports) was accessed from the Haver Analytics database USECON. For the monthiy variables, we select a dataset of $N=62$ predictors from the FRED-MD database, all transiormed to stationary using the transformations recommended by McCracken and Ng (2016). A fuil description of these variables, along with information regarding their publication lag, can be found in the Supplementary Material. The variables were selected to be similar to the "medium" case of Bańbura and Modugno (2014) who show that factors estimated from the largest form of the Stock and Watson-type dataset, like FRED-MD, tend to perform worse than smaller datasets in nowcasting GDP as they contain too many price and financial series. Our database mostly contains data on 


\section{ACCEPTED MANUSCRIPT}

production, surveys, employment, prices and housing series. This dataset is somewhat larger than many existing factor model nowcasting studies for the U.S. such as Antolín Díaz et al. (2017).

Regarding the timeliness of the data flow, the first release of the target GDP series occurs just under a month after the end of the reference quarter, for example Q1 is released near the end of April. The most timely monthly data is the consumer sentiment index which the only variable to be released before the end of the observation month. This is shortly followed by the financial series which are available without lag as they are aggregated from daily data. Employment data are also very timely, whereas industrial production, housing and loans variables are released some weeks after the ena of the observation month.

This information is used to generate a calendar of releases which deternines the sequence of nowcasts we use to predict each quarter's GDP growth. Starting from the first day of the quarter and finishing around 118 days later when GDP is released, we consider every ynique release of data. This includes cases where data are released at different times in the same day, such is the case with the retail sales and industrial production variables. Counting up the distinct releases in the 3 months of the nowcast period and the 28 days of the backcast period before GDP is released gives a total of $S=53$ different nowcast updates per quarter.

We use data on all months and quarters between 1978Q2 and 2016Q1, which is the common available sample for all variables. This gives a total of $T=152$ quarterly observations and $3 T=456$ monthly observations. We use the ecursive out-of-sample method described in Section 2.2 with different splits of $R$ and $P$, namely $(R, P) \in\{(101,51),(87,65),(76,76),(65,87),(51,101)\}$ which correspond to ratios of $P / R \in\{1,2,3 / 4,1,4 / 3,2\}$.

At every prediction boint $i=R+1, \ldots, T$ and at every nowcast point $i=1, \ldots, S$, in the first step we solve the ragged edge problem using a simple $A R(1)$ interpolation as in Kim and Swanson (2017). We then estimate the factors by PCA as in Equation (6). We estimate the number of factors $r$ using the $I C_{\gamma 2}$ information criterion of Bai and $\mathrm{Ng}(2002$ based on the first window of data for the case $P / R=1$ and fix this number throughout the out-of-sample experiment. This results in $r=4$ factors which is between the $r=8$ factors found by McCracken and $\mathrm{Ng}(2016)$ on the full set of $N=134$ FRED-MD variables, and $r=1$ used in the small-scale factor model of Antolín Díaz et al. (2017).

We make nowcasts using the quarterly regression model:

$$
y_{t}=\mu+\rho y_{t-1}+\beta_{0}^{\prime} F_{t}+\beta_{1}^{\prime} F_{t-1 / 3}+\beta_{2}^{\prime} F_{t-2 / 3}+\varepsilon_{t}
$$

which is equivalent to Equation (3) with $W_{t}=\left[1, y_{t-1}\right]^{\prime}$, so the must-have regressors $W_{t}$ are just a 


\section{ACCEPTED MANUSCRIPT}

constant and a lag of $y_{t}$. Since the lagged quarter of GDP is not available in the first 28 days of the nowcast period, as with the monthly variables we solve the ragged edge by substituting $y_{t-1}$ with the prediction from an $A R(1)$ model until the data point is released.

In generating the sets of moment inequalities for our test, we measure nowcast error losses using MSFE. We first consider using the full set of all $S(S-1) / 2$ pairwise moment inequalities, which gives a high-dimensional set-up with $\kappa=1378$. As in the Monte Carlo section, we will compare our resalts to the adjacent-only version of the test, similar to the $\boldsymbol{\Delta}^{\mathrm{e}}$ test of Patton and Timmermann (2012), and the version with inequality spacing of $k_{S}^{c}=5$ (we checked the results for $k_{S}^{c} \in\{1,2\}$ which were very similar and therefore omitted). We determine the block lengths using the optimal procedure outlined in the Supplementary Material and generate $B=399$ bootstrap draws.

We also ran other configurations to explore the robustness of the empirical results and assess how the test performs. Firstly, the rolling estimation scheme is used in place of the recursive scheme. Next, we consider a reduced dataset of $N=9$, similar to the "small" case of Baribura and Modugno (2014), to observe any differences resulting from dataset composition We alsu explore grouping data releases together into days (not updating nowcasts within the day) and also in sets of 10 days, rather than using each unique release timing. We next explore the sensitivity to the date span used by providing results with a post-1984 sample, motivated by evidence such as Breitung and Eickmeier (2011) who find a break in the factor loadings in around 1984, attributed to the 'Great Moderation'. Finally, we check the results when the asymmetric Linex loss function is used instead of squared error loss.

\subsection{Results}

The graphs in Figure/2 iepret the evolution of MSFE for the aggregate real GDP growth variable and the five GDP sub-components, for the scenario where $(R, P)=(76,76)$. Upon graphical inspection of the results, he fop-1eit panel of Figure 2 shows that the nowcasts for aggregate real GDP growth improve throughout the period, with the quarterly MSFE dropping from $0.35 \%$ to below $0.25 \%$ before flattening in the backcast period.

For consumption, investment, exports and imports the MSFE profiles are also clearly downwardssiening. In some cases there are jumps such as for exports at $i=13$ when the previous quarter's exports data is released, and for investment at $i=16$ when a group of employment-related series are released simultaneously. However in all of these series, including aggregate GDP, there are some minor violations of monotonicity with small segments of rising MSFE. Our test is able to determine whether or not these deviations from monotonicity are statistically significant or are merely features 


\section{ACCEPTED MANUSCRIPT}

Figure 2: MSFE by Nowcast Horizon for the Sample with $(R, P)=(76,76)$
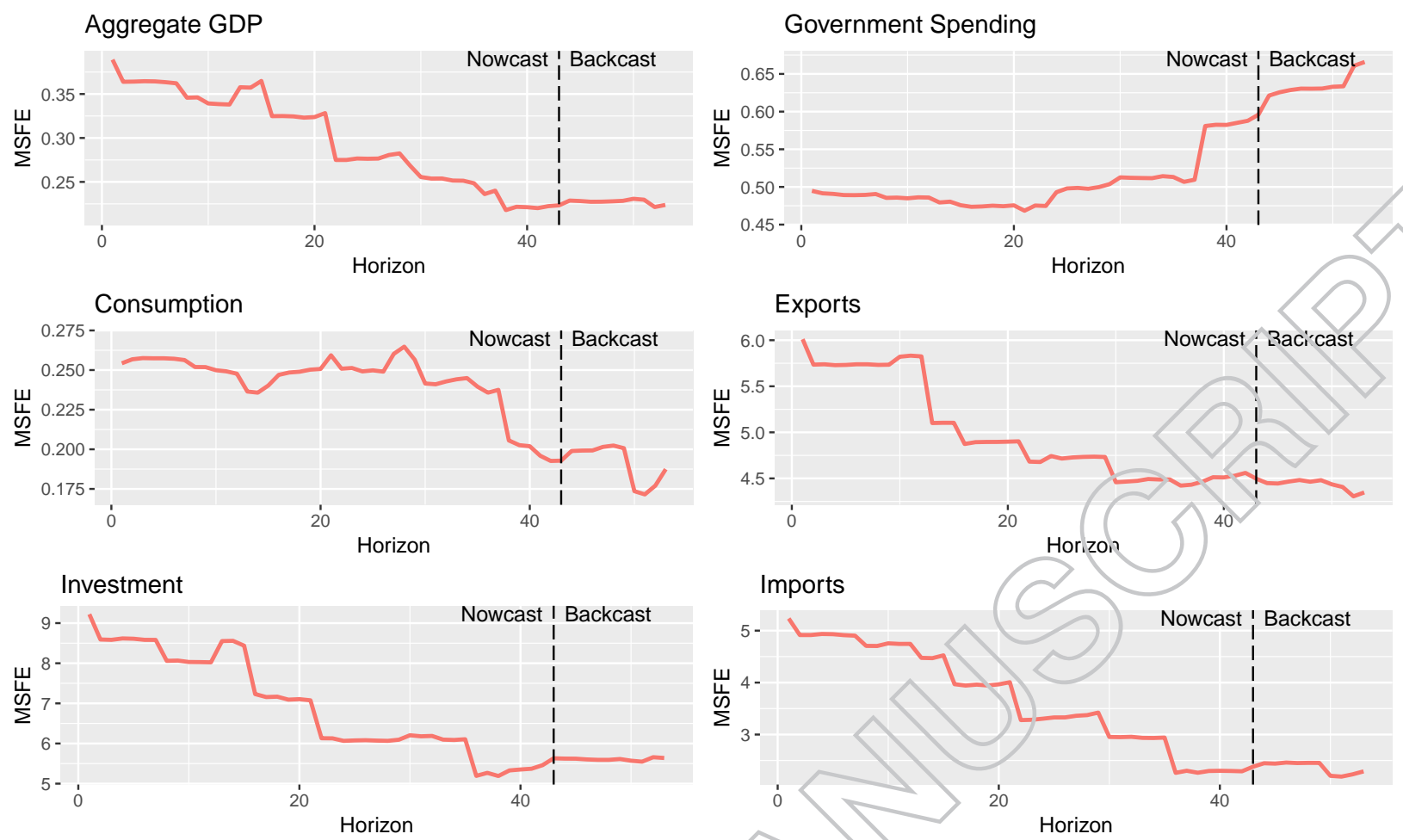

Notes: Horizon refers to the point throughout the prediction period at which we estimate the factor model and forecasting model.

caused by small-sample heterogeneity in the data.

The most striking feature is that of the government spending component, where MSFE is increasing throughout most of the prediction period, from $0.5 \%$ to more than $0.65 \%$. Since this increase seems to be sizeable, we expect our test 60 sect the null hypothesis of monotonicity.

We now turn to the results of our test, which can formally detect whether these MSFE profiles are monotonically declining rather than simply relying only on the graphical evidence in Figure 2, The results are displayed in Table 3, where the first two sets of columns use our test with the full set of inequalities and then with spacing $k_{S}^{c}=5$. The last set of columns uses the adjacent-only comparisons as in the $\Delta^{e}$ test of Patton and Timmermann (2012).

The first thing to note is that the results of our test appear to be very stable, both across the various $(R, P)$ combinations and also as we move from the full set of moment inequalities to the spacing $k_{S}^{c}=5$. For the headline aggregate GDP series, the test finds no evidence of non-monotonicity with $p$-values around 0.7 in these versions of the test. This finding mirrors the result of Bańbura et al. (2013), though using a different method for estimating the factors for the nowcasting model, and over a different in-sample and out-of-sample period. This suggests that factor-based methods are 


\section{ACCEPTED MANUSCRIPT}

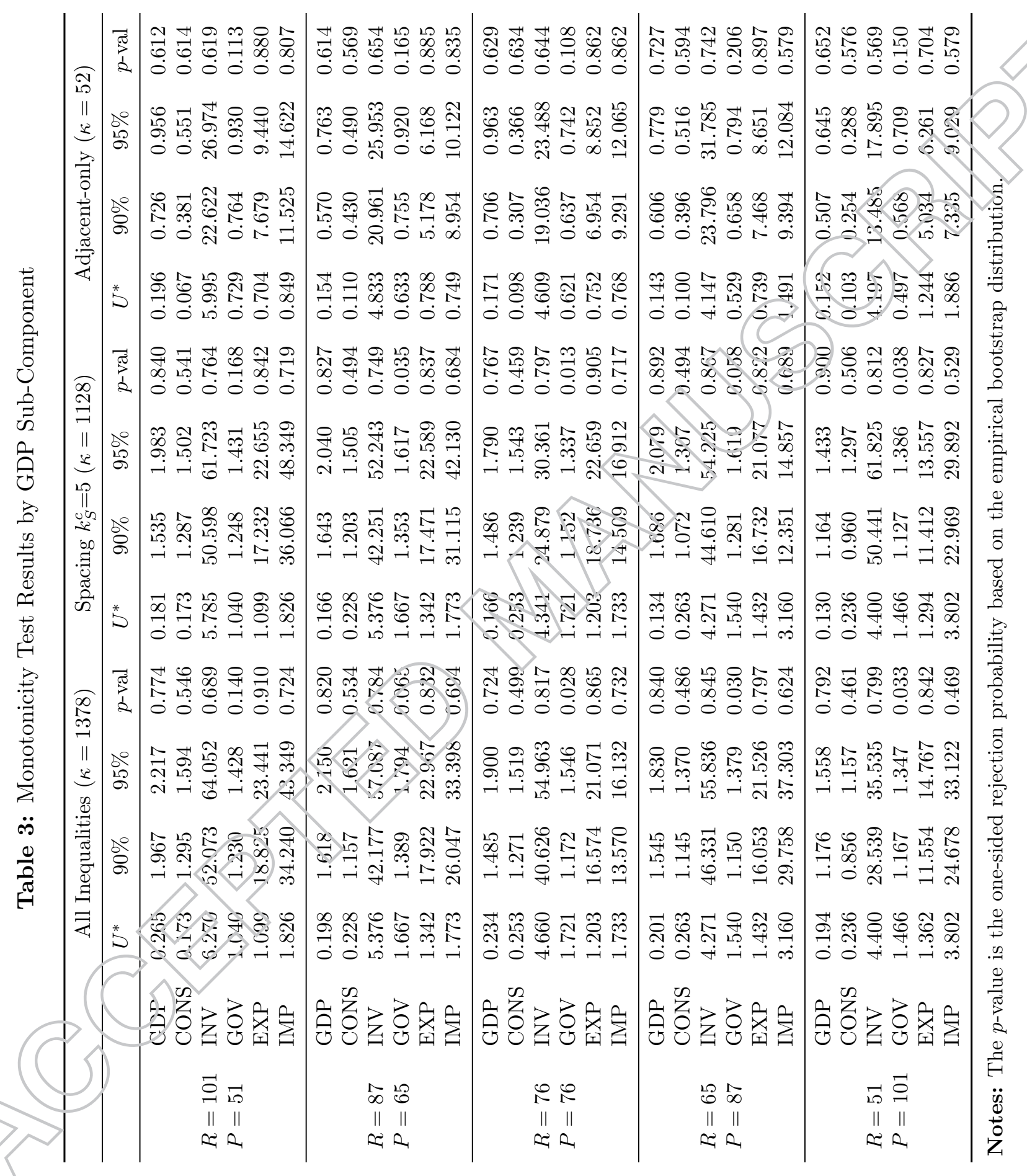




\section{ACCEPTED MANUSCRIPT}

able to generate monotonically improving nowcasts of U.S. real GDP growth irrespective of the factor estimation method or data span. The results for consumption, investment, imports and exports are very similar to the result for aggregate GDP, with large $p$-values as expected.

In the case of government spending the first two versions of the test find significant evidence of non-monotonicity with many rejections at the $5 \%$ level, with the exception of the smallest sample size with $P=51$. This finding shows that our test is able to reject in scenarios where there are clear violations of monotonicity. It is interesting to note that the adjacent-only version of the test, howerer, does not reject the null at the $5 \%$ significance level in any configuration. This aligns with the ressilts of the Monte Carlo simulations where very low power was detected for upward-sloping D.MFE profiles.

Finally, we also find that our results are robust when switching to the rolling estimation scheme and when reducing the dataset to $N=9$. These results, all of which can be fornd in the Supplementary Material, show even stronger rejection of the null in the government spenumg component with many at the $1 \%$ significance level. The adjacent-only version of the test still fojils to reject the null across almost every configuration. The findings are also very similar when we only make $S=12$ nowcasts per quarter by updating the nowcasts every 10 days, even though the maximum number of moment inequalities drops to $\kappa=66$. The idea of using the post-1984 sample motivated by possible structural breaks in the factor loadings also has very minima bearing on the results. Lastly, even when the nowcaster has asymmetric Linex loss with aversion to negative prediction errors, they come to the same conclusion regarding nowcast monotonicity.

\section{Conclusion}

This paper proposes a test te formally assess the performance of 'big data' nowcasting methods. In particular, we focus on models involving estimated factors which have become a leading case in the high-dimensional empirical nowcasting literature, though the test can readily deal with smalldimensional set-ups like bridge equations and MIDAS models. The test uses moment inequalities to evalyate the monotonicity of metrics for nowcast accuracy such as MSFE. Our method differs frem previous forecast accuracy tests not only in being able to accommodate this high-dimensional row casting setting, but also because our interest is in evaluating the nowcasts of a single method at various points in a quarter, rather than comparing two or more models at a single fixed point.

The main contribution of this paper is in extending the methodology of Chernozhukov et al. (2014) for testing many moment inequalities to the case of nowcast monotonicity testing, which allows the number of inequalities to grow with the sample size. We also show that their moment selection pro- 


\section{ACCEPTED MANUSCRIPT}

cedure to rule out non-binding inequalities remains applicable in our context. We then provide the conditions under which both parameter estimation error and factor estimation error are asymptotically negligible in this high dimensional setting when using the pseudo out-of-sample approach of West (1996). We derive conditions required for using factor-augmented models of Stock and Watson (2002a b) and Bai and $\mathrm{Ng}(2006)$ in this nowcast monotonicity test, with the factors estimated by Principal Components Analysis (PCA), after solving the issue of the 'ragged edge'.

We illustrate the finite sample performance of our test through Monte Carlo simulations, and provide an in-depth empirical application to nowcasting the growth rate of U.S. aggregate real GDP, and the growth rate of five GDP subcomponents. Our test confirms that there is no statistical evidence of non-monotonicity in the MSFE of aggregate GDP growth as more information is added throughout the nowcast period. However, the test does pick up violations of monotonicity in the government spending component of GDP. This result suggests that the factor-augmented model may be misspecified for nowcasting government spending, and that alternative models should be considered.

\section{References}

Aastveit, K. A., K. R. Gerdrup, A. S. Jore, and 1. A. Thorsrud (2014). Nowcasting GDP in Real Time: A Density Combination Approach. Jomrnal of Business 83 Economic Statistics 32(1), 48-68.

Altissimo, F., R. Cristadoro, M. Forni, M. Lippi/and G. Veronese (2010). New Eurocoin: Tracking Economic Growth in Real Time. The Review of Economics and Statistics 92(4), 1024-1034.

Antolín Díaz, J., T. Drechsel, and 1. Petrella (2017). Tracking the Slowdown in Long-Run GDP Growth. The Review of Ecconomics and Statistics 99(2), 343-356.

Baffigi, A., R. Golinel1. and G. Parigi (2004). Bridge Models to Forecast the Euro Area GDP. International Jouinal of Forecasting 20(3), 447-460.

Bai, J. and S. No (2002). Determining the Number of Factors in Approximate Factor Models. Econometrica $7 \mathrm{Q}(1), 191-221$.

Bai, J. and S. Ng 2006). Confidence Intervals for Diffusion Index Forecasts and Inference for FactorAugnented Fegressions. Econometrica 74 (4), 1133-1150.

Bańbura Mi., D. Giannone, M. Modugno, and L. Reichlin (2013). Now-Casting and the Real-Time Data F'low. In G. Elliott and A. Timmermann (Eds.), Handbook of Economic Forecasting, Volume 2A, pp. 195-236. Amsterdam: North-Holland.

Bańbura, M. and M. Modugno (2014). Maximum Likelihood Estimation of Factor Models on Datasets with Arbitrary Pattern of Missing Data. Journal of Applied Econometrics 29(1), 133-160.

Bernstein, S. (1927). Sur l'extension du théorème limite du calcul des probabilités aux sommes de quantités dépendantes. Mathematische Annalen 97(1), 1-59.

Bragoli, D. and J. Fosten (2018). Nowcasting Indian GDP. Oxford Bulletin of Economics and Statistics $80(2), 259-282$. 


\section{ACCEPTED MANUSCRIPT}

Breitung, J. and S. Eickmeier (2011). Testing for Structural Breaks in Dynamic Factor Models. Journal of Econometrics 163(1), 71-84.

Bühlmann, P. and H. Künsch (1999). Block Length Selection in the Bootstrap for Time Series. Computational Statistics and Data Analysis 31, 295-310.

Chernozhukov, V., D. Chetverikov, and K. Kato (2014). Testing Many Moment Inequalities. cemmap Working Paper CWP65/13, Massachusetts Institute of Technology.

Clark, T. E. and M. W. McCracken (2009). Improving Forecast Accuracy by Combining Recursive and Rolling Forecasts. International Economic Review 50(2), 363-395.

Clements, M. P. and A. B. Galvão (2008). Macroeconomic Forecasting With Mixed-Frequency Lata. Journal of Business \& Economic Statistics 26(4), 546-554.

Clements, M. P. and A. B. Galvão (2009). Forecasting US Output Growth Using Leacing indicators: an Appraisal Using MIDAS Models. Journal of Applied Econometrics 24(7) 1187-1205.

Corradi, V. and N. R. Swanson (2014). Testing for Structural Stability of Facter A.ugmented Forecasting Models. Journal of Econometrics 182(1), 100-118.

Diebold, F. X. and R. S. Mariano (1995). Comparing Predictive Accuracy Journal of Business and Economic Statistics 13(3), 253-263.

Doz, C., D. Giannone, and L. Reichlin (2011). A Two-Step Estimator for Large Approximate Dynamic Factor Models Based on Kalman Filtering. Journal of Ecorometrics 164 (1), 188-205.

Foroni, C. and M. Marcellino (2014). A Comparison of Nhiyed Frequency Approaches for Nowcasting Euro Area Macroeconomic Aggregates. Internationai Journal of Forecasting 30(3), 554-568.

Fosten, J. (2016). Forecast Evaluation with Factor-Augmented Models. UEA School of Economics Working Paper 2016-05.

Ghosal, S., A. Sen, and A. W. van der Vart (2000). Testing Monotonicity of Regression. Annals of Statistics 28(4), 1054-1092.

Giacomini, R., D. N. Politis, and H. White (2013). A Warp-Speed Method for Conducting Monte Carlo Experiments Inyolving Bootstrap Estimators. Econometric Theory 29(3), 567-589.

Giannone, D., L. Rerchlin, and D. Small (2008). Nowcasting: The Real-time Informational Content of Macroeconomic Data. Journal of Monetary Economics 55(4), 665-676.

Gonçalves, S., IM. T. M.Cracken, and B. Perron (2017). Tests of Equal Accuracy for Nested Models with Estimatec Fartors. Journal of Econometrics 198(2), 231-252.

Hall, P., J. Horowitz, and B. Jing (1995). On Blocking Rules For the Bootstrap with Dependent Data. Biometrika 82, 561-574.

Hansen, P. R. (2005). A Test for Superior Predictive Ability. Journal of Business 85 Economic Stutistics 23(4), 365-380.

Higgins, P. C. (2014). GDPNow: A Model for GDP 'Nowcasting'. FRB Atlanta Working Paper $2014-7$.

Kim, H. H. and N. R. Swanson (2017). Methods for Backcasting, Nowcasting and Forecasting Using Factor-MIDAS: With An Application To Korean GDP. Journal of Forecasting (forthcoming).

Knotek, E. S. and S. Zaman (2017). Nowcasting US Headline and Core Inflation. Journal of Money, Credit and Banking 49(5), 931-968. 


\section{ACCEPTED MANUSCRIPT}

Luciani, M. and L. Ricci (2014). Nowcasting Norway. International Journal of Central Banking 10(4), $215-248$.

Marcellino, M., M. Porqueddu, and F. Venditti (2016). Short-Term GDP Forecasting With a MixedFrequency Dynamic Factor Model With Stochastic Volatility. Journal of Business \& Economic Statistics 34(1), 118-127.

McCracken, M. and S. Ng (2016). FRED-MD: A Monthly Database for Macroeconomic Research. Journal of Business 83 Economic Statistics Economic Statisics 34(4), 574-589.

McCracken, M. W. (2000). Robust Out-of-sample Inference. Journal of Econometrics 99(2), 195223.

Patton, A. J. and A. Timmermann (2012). Forecast Rationality Tests Based on Multi-horizon Bounds. Journal of Business \&3 Economic Statistics 30(1), 1-17.

Schumacher, C. (2016). A Comparison of MIDAS and Bridge Equations. International Iournal of Forecasting 32(2), 257-270.

Stock, J. H. and M. W. Watson (2002a). Forecasting Using Principal Components from a Large Number of Predictors. Journal of the American Statistical Associatibr 97(460), 1167-1179.

Stock, J. H. and M. W. Watson (2002b). Macroeconomic Forecasting Using Diffusion Indexes. Journal of Business and Economic Statistics 20(2), 147-162.

West, K. D. (1996). Asymptotic Inference about Predictive Äbirity. Econometrica 64(5), 1067-1084.

White, H. (2000). A Reality Check for Data Snooping. Econemetrica 68(5), 1097-1126.

Zhang, X. and G. Cheng (2014). Bootstrapping High Dimensional Time Series. Working paper, University of Missouri-Columbia. 